\title{
Mapping Chemical Respiratory Sensitization: How Useful Are Our Current Computational Tools?
}

Emily Golden ${ }^{1}$, Mikhail Maertens ${ }^{1}$, Thomas Hartung ${ }^{1,2}$, Alexandra Maertens ${ }^{1 *}$

${ }^{1}$ Center for Alternatives to Animal Testing (CAAT), Johns Hopkins Bloomberg School of Public Health, Baltimore, MD, USA

${ }^{2}$ CAAT-Europe, University of Konstanz, Konstanz, Germany

*Correspondence to: Dr. Alexandra Maertens, Center for Alternatives to Animal Testing (CAAT), Johns Hopkins Bloomberg School of Public Health, Department of Environmental Health and Engineering, 615 N. Wolfe St., W7032, Baltimore, MD 21205, email: amaerte1@jhu.edu 


\section{Supplemental Information}

\begin{tabular}{|l|l|}
\hline$\# 1$ & "Organic chemicals"[Mesh] OR "Inorganic chemicals"[Mesh] OR chemical*[tiab] \\
\hline$\# 2$ & $\begin{array}{l}\text { "Occupational Exposure"[Mesh] OR "Inhalation Exposure"[Mesh] OR } \\
\text { "Allergens/chemical synthesis"[Mesh] OR "Allergens/chemistry"[Mesh] OR } \\
\text { "Allergens/toxicity"[Mesh] OR respiratory allergen*[tw] OR respiratory sensitizer*[tw] OR } \\
\text { respiratory sensitiser*[tw] OR inhalational expos*[tw] OR inhalation expos*[tw] OR toxic } \\
\text { inhalation*[tw] OR chemically induced[tw] OR chemical causation[tw] OR chemical cause*[tw] } \\
\text { OR causing chemicals[tw] OR chemical determinant*[tw] OR occupational expos*[tw] OR } \\
\text { chemical respirator*[tw] OR chemical allergen*[tw] OR sensitizing chemical*[tw] OR } \\
\text { sensitising chemical*[tw] }\end{array}$ \\
\hline$\# 3$ & $\begin{array}{l}\text { "Respiratory Hypersensitivity"[Mesh] OR "Alveolitis, Extrinsic Allergic"[Mesh] OR } \\
\text { "Asthma/chemically induced"[Mesh:NoExp] OR "Asthma, Occupational/chemically } \\
\text { induced"[Mesh] OR respiratory hypersensitivity[tw] OR occupational asthma[tw] OR } \\
\text { hypersensitivity pneumonit*[tw] OR Extrinsic Allergic Alveolit*[tw] OR respiratory } \\
\text { sensitization*[tw] OR respiratory sensitisation*[tw] OR occupational asthma*[tw] OR workplace } \\
\text { asthma*[tw] OR respiratory allerg*[tw] }\end{array}$ \\
\hline$\# 4$ & $\begin{array}{l}\text { \#1 AND \#2 AND \#3 } \\
\text { animals [Mesh] NOT humans [Mesh] }\end{array}$ \\
\hline$\# 5$ & \#4 NOT \#5 \\
\hline
\end{tabular}

Table S1: Scoping Search Strategy. Supplemental Table 1 contains search terms included in our scoping search strategy. 


\begin{tabular}{|c|c|c|c|c|c|}
\hline Number & CID & CASRN & Chemical Name & $\begin{array}{l}\text { Sensitization } \\
\text { Status }\end{array}$ & Reference(s) \\
\hline 1 & 18503 & $3173-72-6$ & $\begin{array}{l}\text { 1,5-Naphthalene } \\
\text { diisocyanate }\end{array}$ & Sensitizer & $\begin{array}{c}\text { Graham et al. 1997; } \\
\text { Jarvis et al. 2005; } \\
\text { Enoch et al. 2012; } \\
\text { Seed et al. 2015 }\end{array}$ \\
\hline 2 & 11443 & $584-84-9$ & 2,4-Toluene diisocyanate & Sensitizer & $\begin{array}{l}\text { Graham et al. 1997; } \\
\text { Jarvis et al. 2005; } \\
\text { Lalko et al. 2012; } \\
\text { Seed et al. 2015; } \\
\text { HSDB }\end{array}$ \\
\hline 3 & 7040 & 91-08-7 & 2,6-Toluene diisocyanate & Sensitizer & $\begin{array}{c}\text { Graham et al. 1997; } \\
\text { Enoch et al. 2012; } \\
\text { Lalko et al. 2012; } \\
\text { HSDB }\end{array}$ \\
\hline 4 & 11082 & $551-16-6$ & 6-Amino penicillanic acid & Sensitizer & $\begin{array}{c}\text { Graham et al. 1997; } \\
\text { Jarvis et al. 2005; } \\
\text { Enoch et al. 2012; } \\
\text { Seed et al. } 2015\end{array}$ \\
\hline 5 & 6249 & $69-53-4$ & Ampicillin & Sensitizer & $\begin{array}{c}\text { Graham et al. 1997; } \\
\text { Enoch et al. } 2012\end{array}$ \\
\hline 6 & 5904 & $61-33-6$ & Benzylpenicillin & Sensitizer & $\begin{array}{l}\text { Graham et al. 1997; } \\
\text { Enoch et al. } 2012\end{array}$ \\
\hline 7 & 78141 & $4424-06-0$ & Brilliant orange GR & Sensitizer & Graham et al. 1997 \\
\hline 8 & 10255083 & $1260-17-9$ & Carminic acid & Sensitizer & Graham et al. 1997 \\
\hline 9 & 27447 & $15686-71-2$ & Cephalexin & Sensitizer & $\begin{array}{c}\text { Graham et al. 1997; } \\
\text { Jarvis et al. 2005; } \\
\text { Enoch et al. 2012; } \\
\text { Seed et al. } 2015\end{array}$ \\
\hline 10 & 2713 & $55-56-1$ & Chlorhexidene & Sensitizer & $\begin{array}{l}\text { Graham et al. 1997; } \\
\text { Jarvis et al. 2005; } \\
\text { Enoch et al. 2012; } \\
\text { Lalko et al. 2012; } \\
\text { Seed et al. 2015 }\end{array}$ \\
\hline 11 & 3039 & $62-73-7$ & Dichlorvos & Sensitizer & Graham et al. 1997 \\
\hline
\end{tabular}




\begin{tabular}{|c|c|c|c|c|c|}
\hline Number & CID & CASRN & Chemical Name & $\begin{array}{l}\text { Sensitization } \\
\text { Status }\end{array}$ & Reference(s) \\
\hline 12 & 7902 & $108-01-0$ & Dimethyl ethanolamine & Sensitizer & $\begin{array}{c}\text { Graham et al. 1997; } \\
\text { Jarvis et al. 2005; } \\
\text { Enoch et al. 2012; } \\
\text { Seed et al. 2015 }\end{array}$ \\
\hline 13 & 7570 & $101-68-8$ & $\begin{array}{c}\text { Diphenyl methane- } 4,4 *- \\
\text { diisocyanate }\end{array}$ & Sensitizer & $\begin{array}{l}\text { Graham et al. 1997; } \\
\text { Jarvis et al. 2005; } \\
\text { Lalko et al. 2012; } \\
\text { Seed et al. 2015; } \\
\text { HSDB }\end{array}$ \\
\hline 14 & 65064 & $989-51-5$ & Epigallocatechin gallate & Sensitizer & $\begin{array}{l}\text { Graham et al. 1997; } \\
\text { Enoch et al. 2012; } \\
\text { Seed et al. } 2015\end{array}$ \\
\hline 15 & 700 & $141-43-5$ & Ethanolamine & Sensitizer & $\begin{array}{l}\text { Graham et al. 1997; } \\
\text { Jarvis et al. 2005; } \\
\text { Enoch et al. 2012; } \\
\text { Seed et al. 2015 }\end{array}$ \\
\hline 16 & 81530 & $7085-85-0$ & Ethyl cyanoacrylate & Sensitizer & $\begin{array}{l}\text { Graham et al. 1997; } \\
\text { Jarvis et al. 2005; } \\
\text { Enoch et al. 2012; } \\
\text { Seed et al. 2015 }\end{array}$ \\
\hline 17 & 3301 & $107-15-3$ & Ethylenediamine & Sensitizer & $\begin{array}{c}\text { Graham et al. 1997; } \\
\text { Jarvis et al. 2005; } \\
\text { Enoch et al. 2012; } \\
\text { Lalko et al. } 2012 \\
\text { Seed et al. } 2015\end{array}$ \\
\hline 18 & 3346 & $55-38-9$ & Fenthion & Sensitizer & $\begin{array}{c}\text { Graham et al. 1997; } \\
\text { Jarvis et al. 2005; } \\
\text { Enoch et al. 2012; } \\
\text { Seed et al. 2015 }\end{array}$ \\
\hline 19 & 13192 & $822-06-0$ & $\begin{array}{l}\text { Hexamethylene } \\
\text { diisocyanate }\end{array}$ & Sensitizer & $\begin{array}{c}\text { Graham et al. 1997; } \\
\text { Jarvis et al. 2005; } \\
\text { Enoch et al. 2012; } \\
\text { Lalko et al. 2012; } \\
\text { Seed et al. 2015; } \\
\text { HSDB }\end{array}$ \\
\hline
\end{tabular}




\begin{tabular}{|c|c|c|c|c|c|}
\hline Number & CID & CASRN & Chemical Name & $\begin{array}{c}\text { Sensitization } \\
\text { Status }\end{array}$ & Reference(s) \\
\hline 20 & 169132 & $4098-71-9$ & Isophorone diisocyanate & Sensitizer & $\begin{array}{c}\text { Graham et al. 1997; } \\
\text { Jarvis et al. 2005; } \\
\text { Enoch et al. 2012; } \\
\text { Seed et al. 2015; } \\
\text { HSDB }\end{array}$ \\
\hline 21 & 7923 & $108-31-6$ & Maleic anhydride & Sensitizer & $\begin{array}{l}\text { Graham et al. 1997; } \\
\text { Jarvis et al. 2005; } \\
\text { Enoch et al. 2012; } \\
\text { Lalko et al. 2012; } \\
\text { Seed et al. 2015; }\end{array}$ \\
\hline 22 & 8711 & $137-05-3$ & Methyl-2-cyanoacrylate & Sensitizer & $\begin{array}{c}\text { Graham et al. 1997; } \\
\text { Jarvis et al. 2005; } \\
\text { Enoch et al. 2012; } \\
\text { Seed et al. 2015; }\end{array}$ \\
\hline 23 & 38853 & $555-30-6$ & Methyldopa & Sensitizer & $\begin{array}{l}\text { Graham et al. 1997; } \\
\text { Jarvis et al. 2005; } \\
\text { Enoch et al. 2012; } \\
\text { Seed et al. 2015; }\end{array}$ \\
\hline 24 & 6811 & $85-44-9$ & Phthalic anhydride & Sensitizer & $\begin{array}{c}\text { Graham et al. 1997; } \\
\text { Jarvis et al. 2005; } \\
\text { Enoch et al. 2012; } \\
\text { Lalko et al. 2012; } \\
\text { Seed et al. 2015; } \\
\text { HSDB }\end{array}$ \\
\hline 25 & 43672 & $61477-96-1$ & Piperacillin & Sensitizer & $\begin{array}{l}\text { Graham et al. 1997; } \\
\text { Enoch et al. 2012 }\end{array}$ \\
\hline 26 & 4837 & $110-85-0$ & Piperazine & Sensitizer & $\begin{array}{l}\text { Graham et al. 1997; } \\
\text { Jarvis et al. 2005; } \\
\text { Enoch et al. 2012; } \\
\text { Lalko et al. 2012; } \\
\text { Seed et al. 2015 }\end{array}$ \\
\hline 27 & 104836 & $16462-65-0$ & Plicatic acid & Sensitizer & $\begin{array}{l}\text { Graham et al. 1997; } \\
\text { Enoch et al. 2012; } \\
\text { Seed et al. 2015 }\end{array}$ \\
\hline 28 & 15910 & $1897-45-6$ & Tetrachloroisophthalonitrile & Sensitizer & $\begin{array}{l}\text { Graham et al. 1997; } \\
\text { Jarvis et al. 2005; } \\
\text { Seed et al. 2015 }\end{array}$ \\
\hline
\end{tabular}




\begin{tabular}{|c|c|c|c|c|c|}
\hline Number & CID & CASRN & Chemical Name & $\begin{array}{l}\text { Sensitization } \\
\text { Status }\end{array}$ & Reference(s) \\
\hline 29 & 8326 & $117-08-8$ & $\begin{array}{l}\text { Tetrachlorophthalic } \\
\text { anhydride }\end{array}$ & Sensitizer & $\begin{array}{c}\text { Graham et al. 1997; } \\
\text { Jarvis et al. 2005; } \\
\text { Enoch et al. 2012; } \\
\text { Lalko et al. 2012; } \\
\text { Seed et al. } 2015\end{array}$ \\
\hline 30 & 5565 & $112-24-3$ & Triethylenetetramine & Sensitizer & $\begin{array}{c}\text { Graham et al. 1997; } \\
\text { Jarvis et al. 2005; } \\
\text { Enoch et al. 2012; } \\
\text { Seed et al. } 2015\end{array}$ \\
\hline 31 & 11089 & $552-30-7$ & Trimellitic anhydride & Sensitizer & $\begin{array}{c}\text { Graham et al. 1997; } \\
\text { Jarvis et al. 2005; } \\
\text { Enoch et al. 2012; } \\
\text { Lalko et al. 2012; } \\
\text { Seed et al. 2015; } \\
\text { HSDB }\end{array}$ \\
\hline 32 & 5280440 & $1401-69-0$ & Tylosin & Sensitizer & $\begin{array}{l}\text { Graham et al. 1997; } \\
\text { Enoch et al. } 2012\end{array}$ \\
\hline 33 & 67005 & $116-66-5$ & $\begin{array}{c}\text { 1,1,3,3,5-Pentamethyl-4,6- } \\
\text { Dinitroindane }\end{array}$ & Non-sensitizer & Graham et al. 1997 \\
\hline 34 & $\mathrm{n} / \mathrm{a}$ & $470-67-7$ & 1,4-Cineole & Non-sensitizer & Graham et al. 1997 \\
\hline 35 & 8103 & $111-27-3$ & 1-Hexanol & Non-sensitizer & Graham et al. 1997 \\
\hline 36 & 94602 & $62346-96-7$ & $\begin{array}{l}\text { 2,4-Dimethyl benzyl } \\
\text { acetate }\end{array}$ & Non-sensitizer & Graham et al. 1997 \\
\hline 37 & 94569 & $54546-26-8$ & $\begin{array}{c}\text { 2-Butyl-4,4,6-trimethyl- } \\
\text { 1,3-dioxane }\end{array}$ & Non-sensitizer & Graham et al. 1997 \\
\hline 38 & 62240 & $67874-72-0$ & $\begin{array}{l}\text { 2-tert-Amylcyclohexyl } \\
\text { acetate }\end{array}$ & Non-sensitizer & Graham et al. 1997 \\
\hline 39 & 108506 & $60763-42-0$ & $\begin{array}{c}\text { 3,6-Dimethyloctan- } \\
\text { 3-yl acetate }\end{array}$ & Non-sensitizer & Graham et al. 1997 \\
\hline 40 & 61361 & $6066-49-5$ & 3-Butyl phthalide & Non-sensitizer & Graham et al. 1997 \\
\hline 41 & 61585 & $13171-00-1$ & $\begin{array}{l}\text { 4-Acetyl-6-tert-butyl-1,1- } \\
\text { dimethylindane }\end{array}$ & Non-sensitizer & Graham et al. 1997 \\
\hline 42 & 39801 & 50816-18-7 & 9-Decenyl acetate & Non-sensitizer & Graham et al. 1997 \\
\hline 43 & 6930 & $88-29-9$ & $\begin{array}{l}\text { Acetyl } \\
\text { ethyltetramethyltetralin }\end{array}$ & Non-sensitizer & Graham et al. 1997 \\
\hline
\end{tabular}




\begin{tabular}{|c|c|c|c|c|c|}
\hline Number & CID & CASRN & Chemical Name & $\begin{array}{c}\text { Sensitization } \\
\text { Status }\end{array}$ & Reference(s) \\
\hline 44 & 8878 & $142-19-8$ & Allyl heptylate & Non-sensitizer & Graham et al. 1997 \\
\hline 45 & 7650 & $103-37-7$ & Benzyl butyrate & Non-sensitizer & Graham et al. 1997 \\
\hline 46 & 7353 & $97-87-0$ & Butyl isobutyrate & Non-sensitizer & Graham et al. 1997 \\
\hline 47 & 6616 & $79-92-5$ & Camphene & Non-sensitizer & Graham et al. 1997 \\
\hline 48 & 5372353 & $65405-76-7$ & cis-3-Hexenyl anthranilate & Non-sensitizer & Graham et al. 1997 \\
\hline 49 & 5362620 & 21662-09-9 & cis-4-Decen-1-al & Non-sensitizer & Graham et al. 1997 \\
\hline 50 & 40058 & $52671-32-6$ & Citronellyl nitrile & Non-sensitizer & Graham et al. 1997 \\
\hline 51 & 20508 & $4442-79-9$ & Cyclohexylethyl alcohol & Non-sensitizer & Graham et al. 1997 \\
\hline 52 & 11002 & $544-40-1$ & Dibutyl sulfide & Non-sensitizer & Graham et al. 1997 \\
\hline 53 & 66768 & $95-41-0$ & Dihydro-isojasmone & Non-sensitizer & Graham et al. 1997 \\
\hline 54 & 80865 & $6413-10-1$ & $\begin{array}{l}\text { Ethyl acetoacetate } \\
\text { ethylene glycol ketal }\end{array}$ & Non-sensitizer & Graham et al. 1997 \\
\hline 55 & 7344 & $97-64-3$ & Ethyl lactate & Non-sensitizer & Graham et al. 1997 \\
\hline 56 & 25238 & $10402-33-2$ & Eugenyl phenylacetate & Non-sensitizer & Graham et al. 1997 \\
\hline 57 & 16821 & $2305-05-7$ & g-Dodecalactone & Non-sensitizer & Graham et al. 1997 \\
\hline 58 & 5353011 & $94-48-4$ & Geranyl benzoate & Non-sensitizer & Graham et al. 1997 \\
\hline 59 & 62592 & 5870-93-9 & Heptyl butyrate & Non-sensitizer & Graham et al. 1997 \\
\hline 60 & 8058 & $110-54-3$ & Hexane & Non-sensitizer & Graham et al. 1997 \\
\hline 61 & 637523 & $16930-96-4$ & Hexyl tiglate & Non-sensitizer & Graham et al. 1997 \\
\hline 62 & 7795 & $106-27-4$ & Isoamyl butyrate & Non-sensitizer & Graham et al. 1997 \\
\hline 63 & 7336 & $97-45-0$ & 1-Carvyl propionate & Non-sensitizer & Graham et al. 1997 \\
\hline 64 & 89479 & $21280-29-5$ & Lactoscatone & Non-sensitizer & Graham et al. 1997 \\
\hline 65 & 5323652 & $6622-76-0$ & Methyl tiglate & Non-sensitizer & Graham et al. 1997 \\
\hline 66 & 20739 & 4621-04-9 & p-Isopropylcyclohexanol & Non-sensitizer & Graham et al. 1997 \\
\hline 67 & 62341 & $93-92-5$ & Phenylethyl acetate & Non-sensitizer & Graham et al. 1997 \\
\hline 68 & 61035 & $112-19-6$ & Undecenyl acetate & Non-sensitizer & Graham et al. 1997 \\
\hline 69 & 712 & $50-00-0$ & Formaldehyde & Sensitizer & $\begin{array}{c}\text { Jarvis et al. 2005; } \\
\text { Enoch et al. 2012; } \\
\text { Seed et al. 2015; } \\
\text { HSDB* }\end{array}$ \\
\hline 70 & 5852 & $52-67-5$ & Penicillamine & Sensitizer & $\begin{array}{l}\text { Jarvis et al. 2005; } \\
\text { Enoch et al. 2012; } \\
\text { Seed et al. 2015; }\end{array}$ \\
\hline 71 & 3767 & $54-85-3$ & Isoniazid & Sensitizer & $\begin{array}{l}\text { Jarvis et al. 2005; } \\
\text { Enoch et al. 2012; } \\
\text { Seed et al. 2015; }\end{array}$ \\
\hline
\end{tabular}




\begin{tabular}{|c|c|c|c|c|c|}
\hline Number & CID & CASRN & Chemical Name & $\begin{array}{l}\text { Sensitization } \\
\text { Status }\end{array}$ & Reference(s) \\
\hline 72 & $\mathrm{n} / \mathrm{a}$ & $60-54-8$ & Tetracycline & Sensitizer & $\begin{array}{l}\text { Jarvis et al. 2005; } \\
\text { Enoch et al. 2012; } \\
\text { Seed et al. 2015; }\end{array}$ \\
\hline 73 & 176 & $64-19-7$ & Acetic acid & Sensitizer & $\begin{array}{l}\text { Jarvis et al. 2005; } \\
\text { Enoch et al. 2012; } \\
\text { Seed et al. 2015; }\end{array}$ \\
\hline 74 & 23663979 & $69-52-3$ & Ampicillin & Sensitizer & $\begin{array}{l}\text { Jarvis et al. 2005; } \\
\text { Seed et al. 2015 }\end{array}$ \\
\hline 75 & 23668834 & $69-57-8$ & Benzyl penicillin & Sensitizer & $\begin{array}{l}\text { Jarvis et al. 2005; } \\
\text { Seed et al. 2015 }\end{array}$ \\
\hline 76 & 3598 & $70-30-4$ & Hexachlorophene & Sensitizer & $\begin{array}{l}\text { Jarvis et al. 2005; } \\
\text { Enoch et al. 2012; } \\
\text { Seed et al. 2015; }\end{array}$ \\
\hline 77 & 5340 & $72-14-0$ & Sulfathiazole & Sensitizer & $\begin{array}{l}\text { Jarvis et al. 2005; } \\
\text { Enoch et al. 2012; } \\
\text { Seed et al. 2015; }\end{array}$ \\
\hline 78 & 6354 & $75-21-8$ & Ethylene oxide & Sensitizer & $\begin{array}{l}\text { Jarvis et al. 2005; } \\
\text { Enoch et al. 2012; } \\
\text { Seed et al. 2015; }\end{array}$ \\
\hline 79 & 6658 & $80-62-6$ & Methylmethacrylate & Sensitizer & $\begin{array}{c}\text { Jarvis et al. 2005; } \\
\text { Enoch et al. 2012; } \\
\text { Seed et al. 2015; } \\
\text { HSDB* }\end{array}$ \\
\hline 80 & 85689 & $85-42-7$ & $\begin{array}{l}\text { Hexahydrophthalic } \\
\text { anhydride }\end{array}$ & Sensitizer & $\begin{array}{c}\text { Jarvis et al. 2005; } \\
\text { Enoch et al. 2012; } \\
\text { Lalko et al. 2012; } \\
\text { Seed et al. 2015; } \\
\text { HSDB* }\end{array}$ \\
\hline 81 & 2723 & 88-04-0 & Chloroxylenol & Sensitizer & $\begin{array}{l}\text { Jarvis et al. 2005; } \\
\text { Enoch et al. } 2012\end{array}$ \\
\hline 82 & 6966 & $89-32-7$ & Pyromellitic dianhydride & Sensitizer & $\begin{array}{l}\text { Jarvis et al. 2005; } \\
\text { Seed et al. } 2015\end{array}$ \\
\hline 83 & 3314 & $98-00-0$ & Furfuryl alcohol & Sensitizer & $\begin{array}{l}\text { Jarvis et al. 2005; } \\
\text { Enoch et al. 2012; } \\
\text { Seed et al. 2015; }\end{array}$ \\
\hline 84 & 7361 & $100-37-8$ & 2-Diethylethanolamine & Sensitizer & $\begin{array}{l}\text { Jarvis et al. 2005; } \\
\text { Enoch et al. 2012; } \\
\text { Seed et al. 2015; }\end{array}$ \\
\hline
\end{tabular}




\begin{tabular}{|c|c|c|c|c|c|}
\hline Number & CID & CASRN & Chemical Name & $\begin{array}{l}\text { Sensitization } \\
\quad \text { Status }\end{array}$ & Reference(s) \\
\hline 85 & 7501 & $100-42-5$ & Styrene & Sensitizer & $\begin{array}{l}\text { Jarvis et al. 2005; } \\
\text { Enoch et al. 2012; } \\
\text { Seed et al. 2015; }\end{array}$ \\
\hline 86 & 4101 & $100-97-0$ & Hexamethylene tetramine & Sensitizer & $\begin{array}{l}\text { Jarvis et al. 2005; } \\
\text { Enoch et al. 2012; } \\
\text { Seed et al. 2015; }\end{array}$ \\
\hline 87 & 7814 & $106-50-3$ & Paraphenylene diamine & Sensitizer & $\begin{array}{l}\text { Jarvis et al. 2005; } \\
\text { Enoch et al. 2012; } \\
\text { Seed et al. 2015; }\end{array}$ \\
\hline 88 & 7972 & $109-02-4$ & N-methylmorpholine & Sensitizer & Jarvis et al. 2005 \\
\hline 89 & 7993 & $109-55-7$ & $\begin{array}{c}3- \\
\text { dimethylaminopropylamine }\end{array}$ & Sensitizer & $\begin{array}{l}\text { Jarvis et al. 2005; } \\
\text { Enoch et al. 2012; } \\
\text { Seed et al. 2015; }\end{array}$ \\
\hline 90 & 3485 & $111-30-8$ & Glutaraldehyde & Sensitizer & $\begin{array}{l}\text { Jarvis et al. 2005; } \\
\text { Enoch et al. 2012; } \\
\text { Lalko et al. 2012; } \\
\text { Seed et al. 2015; } \\
\text { HSDB }\end{array}$ \\
\hline 91 & 8112 & $111-41-1$ & Aminoethylethanolamine & Sensitizer & $\begin{array}{l}\text { Jarvis et al. 2005; } \\
\text { Enoch et al. 2012; } \\
\text { Seed et al. 2015 }\end{array}$ \\
\hline 92 & 8343 & $117-81-7$ & Dioctylphthalate & Sensitizer & $\begin{array}{l}\text { Jarvis et al. 2005; } \\
\text { Enoch et al. } 2012\end{array}$ \\
\hline 93 & 73341 & $121-25-5$ & Amprolium hydrochloride & Sensitizer & $\begin{array}{l}\text { Jarvis et al. 2005; } \\
\text { Seed et al. 2015 }\end{array}$ \\
\hline 94 & 785 & $123-31-9$ & Hydroquinone & Sensitizer & $\begin{array}{l}\text { Jarvis et al. 2005; } \\
\text { Enoch et al. 2012; } \\
\text { Seed et al. 2015 }\end{array}$ \\
\hline 95 & 31269 & $123-77-3$ & Azodicarbonamide & Sensitizer & $\begin{array}{l}\text { Jarvis et al. 2005; } \\
\text { Enoch et al. 2012; } \\
\text { Seed et al. 2015 }\end{array}$ \\
\hline 96 & 196 & $124-04-9$ & Adipic acid & Sensitizer & $\begin{array}{l}\text { Jarvis et al. 2005; } \\
\text { Enoch et al. 2012; } \\
\text { Seed et al. 2015 }\end{array}$ \\
\hline 97 & 3641960 & $127-65-1$ & Chloramine- $\mathrm{T}$ & Sensitizer & $\begin{array}{l}\text { Jarvis et al. 2005; } \\
\text { Enoch et al. 2012; } \\
\text { Lalko et al. 2012; } \\
\text { Seed et al. 2015 }\end{array}$ \\
\hline
\end{tabular}




\begin{tabular}{|c|c|c|c|c|c|}
\hline Number & CID & CASRN & Chemical Name & $\begin{array}{l}\text { Sensitization } \\
\text { Status }\end{array}$ & Reference(s) \\
\hline 98 & 3092 & $148-01-6$ & $\begin{array}{l}\text { 2-methyl-3,5- } \\
\text { dinitrobenzamide }\end{array}$ & Sensitizer & $\begin{array}{l}\text { Jarvis et al. 2005; } \\
\text { Enoch et al. 2012; } \\
\text { Seed et al. 2015 }\end{array}$ \\
\hline 99 & 9351 & $304-20-1$ & Hydralazine & Sensitizer & $\begin{array}{l}\text { Jarvis et al. 2005; } \\
\text { Seed et al. 2015 }\end{array}$ \\
\hline 100 & 67539 & $305-80-6$ & $\begin{array}{c}\text { Pauli's Reagent (4- } \\
\text { diazobenzenesulphonic } \\
\text { acid) }\end{array}$ & Sensitizer & $\begin{array}{l}\text { Jarvis et al. 2005; } \\
\text { Enoch et al. 2012; } \\
\text { Seed et al. 2015 }\end{array}$ \\
\hline 101 & 9433 & $317-34-0$ & Aminophylline & Sensitizer & $\begin{array}{l}\text { Jarvis et al. 2005; } \\
\text { Enoch et al. 2012; } \\
\text { Seed et al. 2015 }\end{array}$ \\
\hline 102 & 10569 & $514-10-3$ & Abietic acid & Sensitizer & $\begin{array}{l}\text { Jarvis et al. 2005; } \\
\text { Enoch et al. 2012; } \\
\text { Seed et al. 2015 }\end{array}$ \\
\hline 103 & 5462328 & $561-27-3$ & Diacetyl morphine & Sensitizer & $\begin{array}{l}\text { Jarvis et al. 2005; } \\
\text { Enoch et al. 2012; } \\
\text { Seed et al. 2015 }\end{array}$ \\
\hline 104 & 441328 & $957-68-6$ & $\begin{array}{c}\text { 7-aminocephalosporanic } \\
\text { acid }\end{array}$ & Sensitizer & $\begin{array}{l}\text { Jarvis et al. 2005; } \\
\text { Enoch et al. 2012; } \\
\text { Seed et al. 2015 }\end{array}$ \\
\hline 105 & 637794 & $2746-19-2$ & Himic anhydride & Sensitizer & $\begin{array}{l}\text { Jarvis et al. 2005; } \\
\text { Enoch et al. 2012; } \\
\text { Seed et al. 2015 }\end{array}$ \\
\hline 106 & 16760159 & $2939-80-2$ & Captafol & Sensitizer & $\begin{array}{l}\text { Jarvis et al. 2005; } \\
\text { Seed et al. 2015 }\end{array}$ \\
\hline 107 & 77411 & $3779-63-3$ & $\begin{array}{c}\text { 1,3,5-tris-(6-isocyanato- } \\
\text { hexyl)-, } 1,3,5 \text {.triazinane- } \\
2,4,6 \text {-trione }\end{array}$ & Sensitizer & $\begin{array}{l}\text { Jarvis et al. 2005; } \\
\text { Seed et al. } 2015\end{array}$ \\
\hline 108 & 19950 & $4035-89-6$ & $\begin{array}{c}\text { Biuret of hexamethylene } \\
\text { diisocyanate }\end{array}$ & Sensitizer & $\begin{array}{l}\text { Jarvis et al. 2005; } \\
\text { Seed et al. } 2015\end{array}$ \\
\hline 109 & 442461 & $13466-78-9$ & 3 -carene & Sensitizer & $\begin{array}{l}\text { Jarvis et al. 2005; } \\
\text { Enoch et al. } 2012\end{array}$ \\
\hline 110 & 76956083 & $28983-56-4$ & Methyl blue & Sensitizer & $\begin{array}{l}\text { Jarvis et al. 2005; } \\
\text { Enoch et al. 2012; } \\
\text { Seed et al. 2015 }\end{array}$ \\
\hline 111 & 3035399 & $31330-63-9$ & Tetrazene & Sensitizer & $\begin{array}{l}\text { Jarvis et al. 2005; } \\
\text { Seed et al. 2015 }\end{array}$ \\
\hline
\end{tabular}




\begin{tabular}{|c|c|c|c|c|c|}
\hline Number & CID & CASRN & Chemical Name & $\begin{array}{c}\text { Sensitization } \\
\text { Status }\end{array}$ & Reference(s) \\
\hline 112 & 39859 & $36519-31-0$ & Salbutamol & Sensitizer & $\begin{array}{l}\text { Jarvis et al. 2005; } \\
\text { Seed et al. } 2015\end{array}$ \\
\hline 113 & 170253 & $39878-87-0$ & Phenylglycine acid chloride & Sensitizer & $\begin{array}{l}\text { Jarvis et al. 2005; } \\
\text { Enoch et al. 2012; } \\
\text { Seed et al. } 2015\end{array}$ \\
\hline 114 & 65324 & $49745-95-1$ & Dobutamine $\mathrm{HCl}$ & Sensitizer & $\begin{array}{l}\text { Jarvis et al. 2005; } \\
\text { Enoch et al. } 2012\end{array}$ \\
\hline 115 & 2756 & $51481-61-9$ & Cimetidine & Sensitizer & $\begin{array}{l}\text { Jarvis et al. 2005; } \\
\text { Enoch et al. 2012; } \\
\text { Seed et al. } 2015\end{array}$ \\
\hline 116 & 62883 & $61336-70-7$ & Amoxicillin & Sensitizer & $\begin{array}{l}\text { Jarvis et al. 2005; } \\
\text { Seed et al. } 2015\end{array}$ \\
\hline 117 & 124621 & $\begin{array}{c}123354-92- \\
7\end{array}$ & $\begin{array}{c}\text { Sodium iso- } \\
\text { nonanoyloxybenzene } \\
\text { sulphonate }\end{array}$ & Sensitizer & $\begin{array}{c}\text { Jarvis et al. 2005; } \\
\text { Seed et al. } 2015\end{array}$ \\
\hline 118 & 5464110 & $52-26-6$ & Morphine hydrochloride & Sensitizer & $\begin{array}{l}\text { Jarvis et al. 2005; } \\
\text { Enoch et al. 2012; } \\
\text { Seed et al. } 2015\end{array}$ \\
\hline 119 & 8113 & $111-42-2$ & Diethanolamine & Sensitizer & $\begin{array}{l}\text { Jarvis et al. 2005; } \\
\text { Enoch et al. 2012; } \\
\text { Seed et al. } 2015\end{array}$ \\
\hline 120 & 8265 & $115-27-5$ & Chlorendic anhydride & Sensitizer & $\begin{array}{l}\text { Jarvis et al. 2005; } \\
\text { Enoch et al. 2012; } \\
\text { Seed et al. } 2015\end{array}$ \\
\hline 121 & 10236 & $485-47-2$ & Ninhydrin & Sensitizer & $\begin{array}{l}\text { Jarvis et al. 2005; } \\
\text { Enoch et al. 2012; } \\
\text { Seed et al. } 2015\end{array}$ \\
\hline 122 & $\mathrm{n} / \mathrm{a}$ & $860-22-0$ & Indigotine & Sensitizer & $\begin{array}{l}\text { Jarvis et al. 2005; } \\
\text { Enoch et al. 2012; } \\
\text { Seed et al. } 2015\end{array}$ \\
\hline 123 & 17142 & $2451-62-9$ & Triglycidyl isocyanurate & Sensitizer & $\begin{array}{l}\text { Jarvis et al. 2005; } \\
\text { Enoch et al. 2012; } \\
\text { Seed et al. } 2015\end{array}$ \\
\hline 124 & 17520 & $2634-33-5$ & 1,2-benzisothiazolin-3-one & Sensitizer & $\begin{array}{l}\text { Jarvis et al. 2005; } \\
\text { Enoch et al. 2012; } \\
\text { Seed et al. } 2015\end{array}$ \\
\hline 125 & 83975 & $7696-12-0$ & Tetramethrin & Sensitizer & $\begin{array}{l}\text { Jarvis et al. 2005; } \\
\text { Enoch et al. 2012; } \\
\text { Seed et al. } 2015\end{array}$ \\
\hline
\end{tabular}




\begin{tabular}{|c|c|c|c|c|c|}
\hline Number & CID & CASRN & Chemical Name & $\begin{array}{l}\text { Sensitization } \\
\text { Status }\end{array}$ & Reference(s) \\
\hline 126 & 53172 & $38661-72-2$ & $\begin{array}{l}\text { 1,3-Bis(isocyanatomethyl) } \\
\text { cyclohexane }\end{array}$ & Sensitizer & $\begin{array}{l}\text { Jarvis et al. 2005; } \\
\text { Enoch et al. 2012; } \\
\text { Seed et al. } 2015\end{array}$ \\
\hline 127 & 42008 & $56796-20-4$ & Cefmetazole & Sensitizer & Jarvis et al. 2005 \\
\hline 128 & 23666879 & $59703-84-3$ & Piperacillin & Sensitizer & $\begin{array}{l}\text { Jarvis et al. 2005; } \\
\text { Seed et al. 2015 }\end{array}$ \\
\hline 129 & 94611 & $64265-57-2$ & polyfunctional aziridine & Sensitizer & $\begin{array}{l}\text { Jarvis et al. 2005; } \\
\text { Seed et al. } 2015\end{array}$ \\
\hline 130 & 4212 & $65271-80-9$ & Mitoxanthrone & Sensitizer & $\begin{array}{l}\text { Jarvis et al. 2005; } \\
\text { Enoch et al. 2012; } \\
\text { Seed et al. } 2015\end{array}$ \\
\hline 131 & 47964 & $66592-87-8$ & Cefadroxil & Sensitizer & $\begin{array}{l}\text { Jarvis et al. 2005; } \\
\text { Seed et al. 2015 }\end{array}$ \\
\hline 132 & 5481173 & $72558-82-8$ & Ceftazidime & Sensitizer & $\begin{array}{l}\text { Jarvis et al. 2005; } \\
\text { Enoch et al. 2012; } \\
\text { Seed et al. 2015 }\end{array}$ \\
\hline 133 & 91731 & $79622-59-6$ & Fluazinam & Sensitizer & $\begin{array}{l}\text { Jarvis et al. 2005; } \\
\text { Enoch et al. 2012; } \\
\text { Seed et al. } 2015\end{array}$ \\
\hline 134 & 5362114 & $82547-81-7$ & Cefteram pivoxil & Sensitizer & $\begin{array}{l}\text { Jarvis et al. 2005; } \\
\text { Enoch et al. 2012; } \\
\text { Seed et al. } 2015\end{array}$ \\
\hline 135 & $\mathrm{n} / \mathrm{a}$ & $\begin{array}{c}125700-67- \\
6\end{array}$ & $\begin{array}{l}\text { 2-(1H-benzotriazol-1-yl)- } \\
\text { 1,1,3,3-tetramethyluronium } \\
\text { tetrafluoroborate }\end{array}$ & Sensitizer & $\begin{array}{l}\text { Jarvis et al. 2005; } \\
\text { Seed et al. } 2015\end{array}$ \\
\hline 136 & 93377 & $68123-13-7$ & Basic blue 99 & Sensitizer & Lalko et al. 2012 \\
\hline 137 & 102090420 & $17095-24-8$ & Black GR Reactive Dye & Sensitizer & Lalko et al. 2012 \\
\hline 138 & 7954 & $108-77-0$ & Cyanuric chloride & Sensitizer & Lalko et al. 2012 \\
\hline 139 & 82984 & $12225-83-1$ & Orange $3 R$ reactive dye & Sensitizer & Lalko et al. 2012 \\
\hline 140 & $\mathrm{n} / \mathrm{a}$ & $10343-58-5$ & Reactive yellow & Sensitizer & Lalko et al. 2012 \\
\hline 141 & 77336 & $3740-18-9$ & $\begin{array}{l}\text { 2,4-dichloro-5- } \\
\text { chlorsulfonyl-benzoic acid }\end{array}$ & Sensitizer & $\begin{array}{l}\text { Enoch et al. 2012; } \\
\text { Seed et al. } 2015\end{array}$ \\
\hline 142 & 2723869 & $16691-43-3$ & $\begin{array}{c}\text { 3-amino-5-mercapto- } \\
\text { 1,2,4-triazole }\end{array}$ & Sensitizer & $\begin{array}{l}\text { Enoch et al. 2012; } \\
\text { Seed et al. 2015 }\end{array}$ \\
\hline
\end{tabular}




\begin{tabular}{|c|c|c|c|c|c|}
\hline Number & CID & CASRN & Chemical Name & $\begin{array}{l}\text { Sensitization } \\
\text { Status }\end{array}$ & Reference(s) \\
\hline 143 & 4075 & $89-57-6$ & 5-aminosalicylic acid & Sensitizer & $\begin{array}{l}\text { Enoch et al. 2012; } \\
\text { Seed et al. } 2015\end{array}$ \\
\hline 144 & 6400459 & $37539-03-0$ & $\begin{array}{l}\text { 7-amino-3-thiomethyl-3- } \\
\text { cephalosporanic acid }\end{array}$ & Sensitizer & Seed et al. 2015 \\
\hline 145 & 2286 & $1675-54-3$ & $\begin{array}{l}\text { Diglycidyl ether of } \\
\text { bisphenol A }\end{array}$ & Sensitizer & $\begin{array}{l}\text { Enoch et al. 2012; } \\
\text { Seed et al. 2015 }\end{array}$ \\
\hline 146 & 12736 & $693-23-2$ & Dodecanedioic acid & Sensitizer & $\begin{array}{l}\text { Enoch et al. 2012; } \\
\text { Seed et al. 2015 }\end{array}$ \\
\hline 147 & 12560 & $114-07-8$ & Erythromycin & Sensitizer & Seed et al. 2015 \\
\hline 148 & 90504 & $24447-78-7$ & $\begin{array}{l}\text { Ethoxylated bisphenol A } \\
\text { diacrylate }\end{array}$ & Sensitizer & Seed et al. 2015 \\
\hline 149 & 3314 & $97-53-0$ & Eugenol & Sensitizer & $\begin{array}{l}\text { Enoch et al. 2012; } \\
\text { Seed et al. } 2015\end{array}$ \\
\hline 150 & 188203 & $77430-27-4$ & Glycyl compound & Sensitizer & $\begin{array}{l}\text { Enoch et al. 2012; } \\
\text { Seed et al. } 2015\end{array}$ \\
\hline 151 & 8753 & $139-07-1$ & $\begin{array}{l}\text { Lauryl dimethyl benzyl } \\
\text { ammonium chloride }\end{array}$ & Sensitizer & Seed et al. 2015 \\
\hline 152 & 6438396 & 64761-66-6 & MM22383 & Sensitizer & $\begin{array}{l}\text { Enoch et al. 2012; } \\
\text { Seed et al. } 2015\end{array}$ \\
\hline 153 & 4807 & $643-79-8$ & $\begin{array}{l}\text { Ortho-phthalaldehyde } \\
\text { (1,2-Phthalic } \\
\text { dicarboxaldehyde) } \\
\end{array}$ & Sensitizer & Seed et al. 2015 \\
\hline 154 & 6042 & $59-43-8$ & Thiamine & Sensitizer & $\begin{array}{l}\text { Enoch et al. 2012; } \\
\text { Seed et al. } 2015\end{array}$ \\
\hline 155 & 27200 & $15318-45-3$ & Thiamphenicol & Sensitizer & $\begin{array}{l}\text { Enoch et al. 2012; } \\
\text { Seed et al. } 2015\end{array}$ \\
\hline 156 & 7618 & $102-71-6$ & Triethanolamine & Sensitizer & Seed et al. 2015 \\
\hline 157 & 27423 & $15625-89-5$ & $\begin{array}{l}\text { Trimethylolpropane } \\
\text { triacrylate }\end{array}$ & Sensitizer & $\begin{array}{l}\text { Enoch et al. 2012; } \\
\text { Seed et al. } 2015\end{array}$ \\
\hline 158 & 60196281 & $74610-55-2$ & Tylosin tartrate & Sensitizer & Seed et al. 2015 \\
\hline 159 & 75500 & $2422-91-5$ & $\begin{array}{c}\text { Triphenylmethane } \\
\text { triisocyanate }\end{array}$ & Sensitizer & Seed et al. 2015 \\
\hline 160 & $\mathrm{n} / \mathrm{a}$ & 26471-62-5 & Toluene diisocyanate & Sensitizer & HSDB \\
\hline 161 & 7672 & $103-71-9$ & Phenyl isocyanate & Sensitizer & HSDB \\
\hline
\end{tabular}




\begin{tabular}{|c|c|c|c|c|c|}
\hline Number & CID & CASRN & Chemical Name & $\begin{array}{l}\text { Sensitization } \\
\text { Status }\end{array}$ & Reference(s) \\
\hline 162 & 16760159 & $2425-06-1$ & Captafol & Sensitizer & $\begin{array}{c}\text { Enoch et al. 2012; } \\
\text { HSDB }\end{array}$ \\
\hline 163 & 11167 & $556-61-6$ & Methyl isothiocyanate & Sensitizer & HSDB \\
\hline 164 & 25517 & $12125-02-9$ & Ammonium chloride & Sensitizer & HSDB \\
\hline 165 & 7607 & $102-36-3$ & $\begin{array}{l}\text { 3,4-Dichlorophenyl } \\
\text { isocyanate }\end{array}$ & Sensitizer & HSDB \\
\hline 166 & 7693 & $104-12-1$ & 4-Chlorophenyl isocyanate & Sensitizer & HSDB \\
\hline 167 & 8111 & $111-40-0$ & Diethylenetriamine & Sensitizer & HSDB \\
\hline 168 & 6581 & $79-10-7$ & Acrylic acid & Non-sensitizer & HSDB \\
\hline 169 & 18502 & $3173-53-3$ & Cyclohexylisocyanate & Sensitizer & $\begin{array}{l}\text { Graham et al. 1997; } \\
\text { Jarvis et al. 2005; } \\
\text { Enoch et al. 2012; } \\
\text { Seed et al. 2015; } \\
\text { HSDB }\end{array}$ \\
\hline 170 & 6588 & $79-27-6$ & 1,1,2,2-Tetrabromoethane & Non-sensitizer & Enoch et al. 2012 \\
\hline 171 & 7115 & $92-94-4$ & $1,1^{\prime}: 4^{\prime}, 1^{\prime}$-Terphenyl & Non-sensitizer & Enoch et al. 2012 \\
\hline 172 & 7247 & $95-63-6$ & 1,2,4-Trimethylbenzene & Non-sensitizer & Enoch et al. 2012 \\
\hline 173 & 7839 & $106-93-4$ & 1,2-Dibromoethane & Non-sensitizer & Enoch et al. 2012 \\
\hline 174 & 10707 & $528-29-0$ & 1,2-Dinitrobenzene & Non-sensitizer & Enoch et al. 2012 \\
\hline 175 & 4685 & $106-46-7$ & 1,4-Dichlorobenzene & Non-sensitizer & Enoch et al. 2012 \\
\hline 176 & 7474 & $100-00-5$ & 1-Chloro-4-nitrobenzene & Non-sensitizer & Enoch et al. 2012 \\
\hline 177 & 13387 & $872-50-4$ & 1-Methyl-2-pyrrolidone & Non-sensitizer & Enoch et al. 2012 \\
\hline 178 & 8134 & $111-77-3$ & $\begin{array}{c}2-(2- \\
\text { Methoxyethoxy)ethanol }\end{array}$ & Non-sensitizer & Enoch et al. 2012 \\
\hline 179 & 1486 & $94-75-7$ & $\begin{array}{l}\text { 2,4-Dichlorophenoxyacetic } \\
\text { acid } \\
\end{array}$ & Non-sensitizer & Enoch et al. 2012 \\
\hline 180 & 7958 & $108-83-8$ & 2,6-Dimethylheptan-4-one & Non-sensitizer & Enoch et al. 2012 \\
\hline 181 & 31404 & $128-37-0$ & 2,6-Di-tert-butyl-p-cresol & Non-sensitizer & Enoch et al. 2012 \\
\hline 182 & 10757 & $532-27-4$ & 2-Chloroacetophenone & Non-sensitizer & Enoch et al. 2012 \\
\hline 183 & 62783 & $24468-13-1$ & 2-Ethylhexyl chloroformate & Non-sensitizer & Enoch et al. 2012 \\
\hline 184 & 61249 & 999-61-1 & 2-Hydroxypropyl acrylate & Non-sensitizer & Enoch et al. 2012 \\
\hline 185 & 398 & $79-46-9$ & 2-Nitropropane & Non-sensitizer & Enoch et al. 2012 \\
\hline 186 & 10439 & 504-29-0 & 2-Pyridylamine & Non-sensitizer & Enoch et al. 2012 \\
\hline 187 & 6984 & $89-72-5$ & 2-sec-Butylphenol & Non-sensitizer & Enoch et al. 2012 \\
\hline 188 & 6544 & $78-59-1$ & $\begin{array}{l}\text { 3,5,5-Trimethylcyclohex- } \\
\text { 2-enone } \\
\end{array}$ & Non-sensitizer & Enoch et al. 2012 \\
\hline 189 & 7308 & $96-69-5$ & $\begin{array}{l}\text { 6,6'-Di-tert-butyl-4,4'- } \\
\text { thiodi-m-cresol }\end{array}$ & Non-sensitizer & Enoch et al. 2012 \\
\hline 190 & 180 & $67-64-1$ & Acetone & Non-sensitizer & Enoch et al. 2012 \\
\hline 191 & 7855 & $107-13-1$ & Acrylonitrile & Non-sensitizer & Enoch et al. 2012 \\
\hline
\end{tabular}




\begin{tabular}{|c|c|c|c|c|c|}
\hline Number & CID & CASRN & Chemical Name & $\begin{array}{c}\text { Sensitization } \\
\text { Status }\end{array}$ & Reference(s) \\
\hline 192 & 33613 & $26787-78-0$ & Amoxicillin & Sensitizer & Enoch et al. 2012 \\
\hline 193 & 8732 & $137-88-2$ & Amprolium hydrochloride & Sensitizer & Enoch et al. 2012 \\
\hline 194 & 2347 & $85-68-7$ & Benzyl butyl phthalate & Non-sensitizer & Enoch et al. 2012 \\
\hline 195 & 159055 & $76-22-2$ & Bornan-2-one & Non-sensitizer & Enoch et al. 2012 \\
\hline 196 & 9411 & $314-40-9$ & Bromacil (ISO) & Non-sensitizer & Enoch et al. 2012 \\
\hline 197 & 263 & $71-36-3$ & Butan-1-ol & Non-sensitizer & Enoch et al. 2012 \\
\hline 198 & 18594026 & $133-06-2$ & Captan (ISO) & Non-sensitizer & Enoch et al. 2012 \\
\hline 199 & 47965 & $50370-12-2$ & Cefadroxil & Sensitizer & Enoch et al. 2012 \\
\hline 200 & 33 & $107-20-0$ & Chloroacetaldehyde & Non-sensitizer & Enoch et al. 2012 \\
\hline 201 & 6372 & $75-45-6$ & Chlorodifluoromethane & Non-sensitizer & Enoch et al. 2012 \\
\hline 202 & 2730 & $2921-88-2$ & Chloropyrifos (ISO) & Non-sensitizer & Enoch et al. 2012 \\
\hline 203 & 643833 & $156-59-2$ & cis 1,2-dichloroethylene & Non-sensitizer & Enoch et al. 2012 \\
\hline 204 & 6429 & $76-14-2$ & Cryofluorane(INN) & Non-sensitizer & Enoch et al. 2012 \\
\hline 205 & 7966 & $108-93-0$ & Cyclohexanol & Non-sensitizer & Enoch et al. 2012 \\
\hline 206 & 8560 & $131-17-9$ & Diallyl phthalate & Non-sensitizer & Enoch et al. 2012 \\
\hline 207 & 3026 & $84-74-2$ & Dibutyl phthalate & Non-sensitizer & Enoch et al. 2012 \\
\hline 208 & 6777 & $84-61-7$ & Dicyclohexyl phthalate & Non-sensitizer & Enoch et al. 2012 \\
\hline 209 & 6781 & $84-66-2$ & Diethyl phthalate & Non-sensitizer & Enoch et al. 2012 \\
\hline 210 & 6163 & $64-67-5$ & Diethyl sulphate & Non-sensitizer & Enoch et al. 2012 \\
\hline 211 & 6782 & $84-69-5$ & Diisobutyl phthalate & Non-sensitizer & Enoch et al. 2012 \\
\hline 212 & 33599 & $26761-40-0$ & Diisodecyl phthalate & Non-sensitizer & Enoch et al. 2012 \\
\hline 213 & 3018042 & $28553-12-0$ & Diisononyl phthalate & Non-sensitizer & Enoch et al. 2012 \\
\hline 214 & 33934 & $27554-26-3$ & Diisooctyl phthalate & Non-sensitizer & Enoch et al. 2012 \\
\hline 215 & 8554 & $131-11-3$ & Dimethyl phthalate & Non-sensitizer & Enoch et al. 2012 \\
\hline 216 & 6787 & $84-76-4$ & Dinonyl phthalate & Non-sensitizer & Enoch et al. 2012 \\
\hline 217 & 7583 & $101-84-8$ & Diphenyl ether & Non-sensitizer & Enoch et al. 2012 \\
\hline 218 & 11487 & $122-39-4$ & Diphenylamine & Non-sensitizer & Enoch et al. 2012 \\
\hline
\end{tabular}




\begin{tabular}{|c|c|c|c|c|c|}
\hline Number & CID & CASRN & Chemical Name & $\begin{array}{l}\text { Sensitization } \\
\text { Status }\end{array}$ & Reference(s) \\
\hline 219 & 7570 & $26447-40-5$ & $\begin{array}{c}\text { Diphenylmethane } \\
\text { di-isocyanate }\end{array}$ & Sensitizer & Enoch et al. 2012 \\
\hline 220 & 3224 & $115-29-7$ & Endosulfan & Non-sensitizer & Enoch et al. 2012 \\
\hline 221 & 702 & $64-17-5$ & Ethanol & Non-sensitizer & Enoch et al. 2012 \\
\hline 222 & 10928 & $541-41-3$ & Ethyl chloroformate & Non-sensitizer & Enoch et al. 2012 \\
\hline 223 & 8025 & $109-94-4$ & Ethyl formate & Non-sensitizer & Enoch et al. 2012 \\
\hline 224 & 3562 & $151-67-7$ & Halothane & Non-sensitizer & Enoch et al. 2012 \\
\hline 225 & 8051 & $110-43-0$ & Heptan-2-one & Non-sensitizer & Enoch et al. 2012 \\
\hline 226 & 11583 & 591-78-6 & Hexan-2-one & Non-sensitizer & Enoch et al. 2012 \\
\hline 227 & 3637 & $86-54-4$ & Hydralazine & Sensitizer & Enoch et al. 2012 \\
\hline 228 & 6556 & 78-78-4 & Isopentane & Non-sensitizer & Enoch et al. 2012 \\
\hline 229 & 4004 & $121-75-5$ & Malathion & Non-sensitizer & Enoch et al. 2012 \\
\hline 230 & 15413 & $1634-04-4$ & Methyl-tert-butyl ether & Non-sensitizer & Enoch et al. 2012 \\
\hline 231 & 198243 & $26590-20-5$ & $\begin{array}{l}\text { Methyltetrahydrophthalic } \\
\text { anhydride }\end{array}$ & Sensitizer & Enoch et al. 2012 \\
\hline 232 & 11723 & $598-56-1$ & N,N-Dimethylethylamine & Non-sensitizer & Enoch et al. 2012 \\
\hline 233 & 10041 & $463-82-1$ & Neopentane & Non-sensitizer & Enoch et al. 2012 \\
\hline 234 & 89594 & $54-11-5$ & Nicotine & Non-sensitizer & Enoch et al. 2012 \\
\hline 235 & 7515 & $100-61-8$ & N-Methylaniline & Non-sensitizer & Enoch et al. 2012 \\
\hline 236 & 971 & $144-62-7$ & Oxalic acid & Non-sensitizer & Enoch et al. 2012 \\
\hline 237 & 4790 & $298-02-2$ & Phorate & Non-sensitizer & Enoch et al. 2012 \\
\hline 238 & 15965 & 1918-02-1 & Picloram (ISO) & Non-sensitizer & Enoch et al. 2012 \\
\hline 239 & 6954 & 88-89-1 & Picric acid & Non-sensitizer & Enoch et al. 2012 \\
\hline 240 & 8893 & $142-64-3$ & Piperazine dihydrochloride & Non-sensitizer & Enoch et al. 2012 \\
\hline 241 & 4944 & 114-26-1 & Propoxur & Non-sensitizer & Enoch et al. 2012 \\
\hline 242 & 4946 & $525-66-6$ & Propranolol & Non-sensitizer & Enoch et al. 2012 \\
\hline 243 & 7397 & $98-59-9$ & $\begin{array}{l}\text { p-Toluenesulphonyl } \\
\text { chloride }\end{array}$ & Non-sensitizer & Enoch et al. 2012 \\
\hline 244 & 289 & $120-80-9$ & Pyrocatechol & Non-sensitizer & Enoch et al. 2012 \\
\hline 245 & 6758 & $83-79-4$ & Rotenone (ISO) & Non-sensitizer & Enoch et al. 2012 \\
\hline 246 & 2083 & 18559-94-9 & Salbutamol & Sensitizer & Enoch et al. 2012 \\
\hline 247 & 23690431 & $136-78-7$ & $\begin{array}{c}\text { Sodium 2-(2,4- } \\
\text { dichlorophenoxy)ethyl } \\
\text { sulphate }\end{array}$ & Non-sensitizer & Enoch et al. 2012 \\
\hline
\end{tabular}




\begin{tabular}{|c|c|c|c|c|c|}
\hline Number & CID & CASRN & Chemical Name & $\begin{array}{l}\text { Sensitization } \\
\text { Status }\end{array}$ & Reference(s) \\
\hline 248 & 5988 & $57-50-1$ & Sucrose & Non-sensitizer & Enoch et al. 2012 \\
\hline 249 & 3032425 & $2422-88-0$ & $\begin{array}{c}\text { 1,1,3-Tributylthiourea } \\
\text { (TBTU) }\end{array}$ & Sensitizer & Enoch et al. 2012 \\
\hline 250 & 31373 & $127-18-4$ & Tetrachloroethylene & Non-sensitizer & Enoch et al. 2012 \\
\hline 251 & 1140 & $108-88-3$ & Toluene & Non-sensitizer & Enoch et al. 2012 \\
\hline 252 & 31357 & $126-73-8$ & Tributyl phosphate & Non-sensitizer & Enoch et al. 2012 \\
\hline 253 & 6423 & $76-06-2$ & Trichloronitromethane & Non-sensitizer & Enoch et al. 2012 \\
\hline 254 & 8376 & $118-96-7$ & Trinitrotoluene & Non-sensitizer & Enoch et al. 2012 \\
\hline 255 & 6527 & $78-30-8$ & Tri-o-tolyl phosphate & Non-sensitizer & Enoch et al. 2012 \\
\hline 256 & 8289 & $115-86-6$ & Triphenyl phosphate & Non-sensitizer & Enoch et al. 2012 \\
\hline 257 & 186099 & $25656-78-4$ & $\begin{array}{l}\text { Triphenylmethane } \\
\text { triisocyanate }\end{array}$ & Sensitizer & Enoch et al. 2012 \\
\hline
\end{tabular}

Table S2: Data Sets. Supplemental Table 2 presents the complete discrete chemical data set. Duplicates have been removed, and chemicals without a usable CASRN or SMILES string were removed. Some chemicals curated from HSDB were classified as non-sensitizers based on available data; however, this sensitization status did not agree with the other references for that chemical (as indicated by an asterisk next to "HSDB" in the References column). Therefore, these chemicals were classified as sensitizers based on a weight-of-evidence approach and to be protective of human health. 

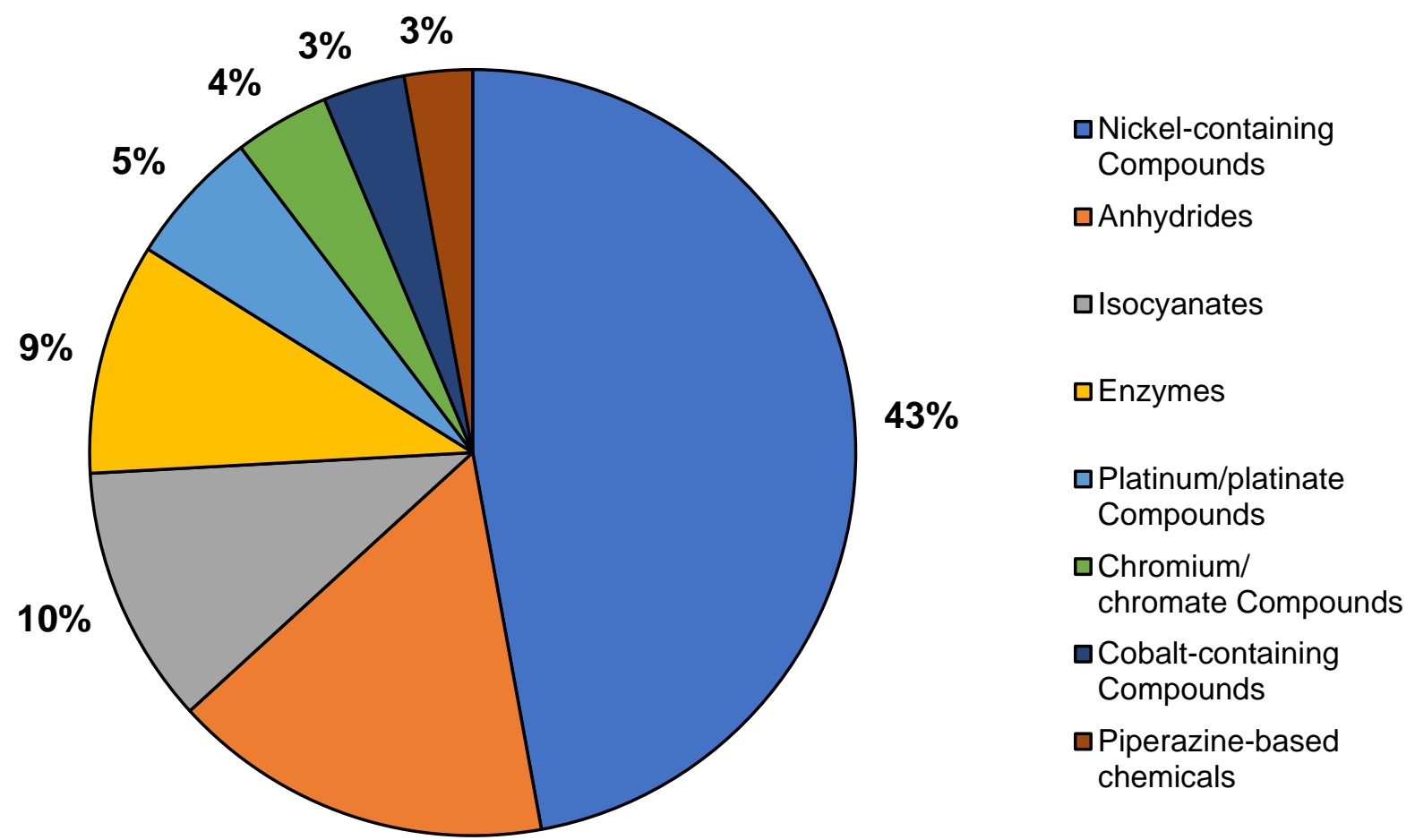

$15 \%$

Figure S1: Harmonized CLP Classification Respiratory Sensitization Chemical Category Distribution. Supplemental Figure 1 presents the harmonized CLP classifications for respiratory sensitization $(n=191)$ by chemical class. Most chemicals with harmonized classifications were metals (55\%) in addition to other well-accepted respiratory sensitizers (anhydrides and isocyanates). 


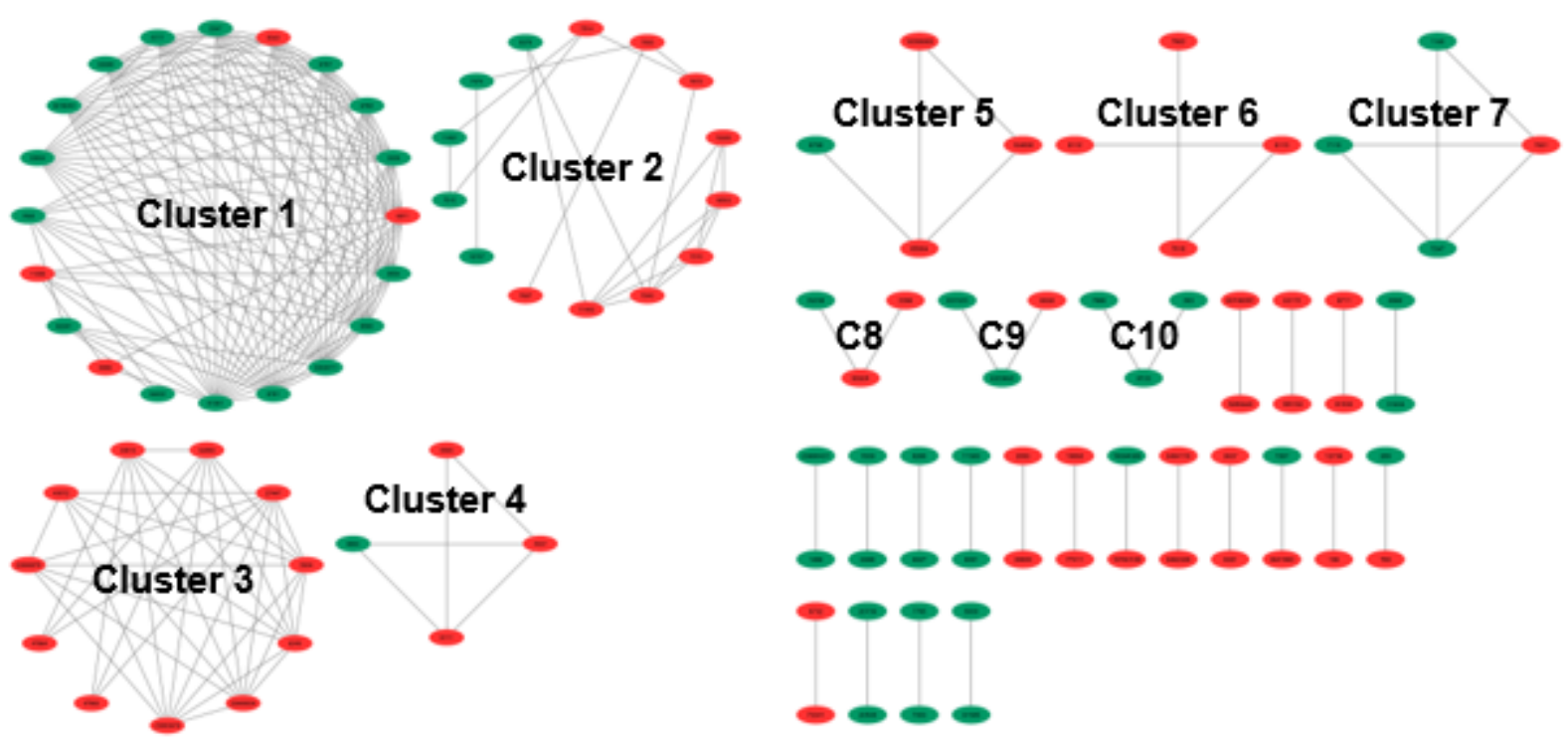

Figure S2: Chemical Similarity Map for the Discrete Chemical Data Set with all Clusters

Identified. Supplemental Figure 2 displays the chemical similarity map (similarity cutoff $=80 \%$ ) for all chemicals in the data set that had at least one neighbor ( $n=110)$ and identifies each cluster with a number. Cluster 8, Cluster 9, and Cluster 10 were abbreviated to C8, C9, and C10, respectively, to adhere to size constraints of the figure. Each chemical in each cluster is identified by CASRN, CID, Chemical name, and sensitization status with the corresponding number of neighbors (using a similarity cutoff of 80\%) in Table S2. 


\begin{tabular}{|c|c|c|c|c|c|c|}
\hline Cluster & $\begin{array}{c}\text { Number } \\
\text { Within } \\
\text { Cluster } \\
\end{array}$ & CASRN & CID & Chemical Name & $\begin{array}{c}\text { Respiratory } \\
\text { Sensitization } \\
\text { Status } \\
\end{array}$ & $\begin{array}{l}\text { Number of } \\
\text { Neighbors }\end{array}$ \\
\hline \multirow{20}{*}{1} & 1 & $84-66-2$ & 6781 & Diethyl phthalate & Non-sensitizer & 17 \\
\hline & 2 & $6066-49-5$ & 61361 & 3-Butyl phthalide & Non-sensitizer & 17 \\
\hline & 3 & $131-11-3$ & 8554 & Dimethyl phthalate & Non-sensitizer & 16 \\
\hline & 4 & $131-17-9$ & 8560 & Diallyl phthalate & Non-sensitizer & 16 \\
\hline & 5 & $94-48-4$ & 5353011 & Geranyl benzoate & Non-sensitizer & 16 \\
\hline & 6 & $85-44-9$ & 6811 & Phthalic anhydride & Sensitizer & 16 \\
\hline & 7 & $84-76-4$ & 6787 & Dinonyl phthalate & Non-sensitizer & 15 \\
\hline & 8 & $84-69-5$ & 6782 & Diisobutyl phthalate & Non-sensitizer & 15 \\
\hline & 9 & $84-74-2$ & 3026 & Dibutyl phthalate & Non-sensitizer & 15 \\
\hline & 10 & $117-81-7$ & 8343 & Dioctylphthalate & Sensitizer & 14 \\
\hline & 11 & $84-61-7$ & 6777 & Dicyclohexyl phthalate & Non-sensitizer & 14 \\
\hline & 12 & $85-68-7$ & 2347 & Benzyl butyl phthalate & Non-sensitizer & 14 \\
\hline & 13 & $\begin{array}{c}\text { 27554-26- } \\
3\end{array}$ & 33934 & Diisooctyl phthalate & Non-sensitizer & 12 \\
\hline & 14 & $\begin{array}{c}28553-12- \\
0\end{array}$ & 3018042 & Diisononyl phthalate & Non-sensitizer & 12 \\
\hline & 15 & $\begin{array}{c}26761-40- \\
0\end{array}$ & 33599 & Diisodecyl phthalate & Non-sensitizer & 12 \\
\hline & 16 & $103-37-7$ & 7650 & Benzyl butyrate & Non-sensitizer & 9 \\
\hline & 17 & $552-30-7$ & 11089 & Trimellitic anhydride & Sensitizer & 6 \\
\hline & 18 & $93-92-5$ & 62341 & Phenylethyl acetate & Non-sensitizer & 5 \\
\hline & 19 & $89-32-7$ & 6966 & Pyromellitic dianhydride & Sensitizer & 3 \\
\hline & 20 & $\begin{array}{c}62346-96- \\
7\end{array}$ & 94602 & 2,4-Dimethyl benzyl acetate & Non-sensitizer & 2 \\
\hline \multirow{14}{*}{2} & 1 & $584-84-9$ & 11443 & 2,4-Toluene diisocyanate & Sensitizer & 5 \\
\hline & 2 & 91-08-7 & 7040 & 2,6-Toluene diisocyanate & Sensitizer & 5 \\
\hline & 3 & $3173-72-6$ & 18503 & 1,5-Naphthalene diisocyanate & Sensitizer & 4 \\
\hline & 4 & $\begin{array}{c}26447-40- \\
5\end{array}$ & 7570 & Diphenylmethane di-isocyanate & Sensitizer & 4 \\
\hline & 5 & $2422-91-5$ & 75500 & Triphenylmethane triisocyanate & Sensitizer & 3 \\
\hline & 6 & $106-50-3$ & 7814 & Paraphenylene diamine & Sensitizer & 3 \\
\hline & 7 & $103-71-9$ & 7672 & Phenyl isocyanate & Sensitizer & 3 \\
\hline & 8 & $104-12-1$ & 7693 & 4-Chlorophenyl isocyanate & Sensitizer & 3 \\
\hline & 9 & $118-96-7$ & 8376 & Trinitrotoluene & Non-sensitizer & 2 \\
\hline & 10 & $122-39-4$ & 11487 & Diphenylamine & Non-sensitizer & 2 \\
\hline & 11 & $100-61-8$ & 7515 & N-Methylaniline & Non-sensitizer & 2 \\
\hline & 12 & $100-00-5$ & 7474 & 1-Chloro-4-nitrobenzene & Non-sensitizer & 2 \\
\hline & 13 & $102-36-3$ & 7607 & 3,4-Dichlorophenyl isocyanate & Sensitizer & 1 \\
\hline & 14 & $528-29-0$ & 10707 & 1,2-Dinitrobenzene & Non-sensitizer & 1 \\
\hline \multirow{4}{*}{3} & 1 & $69-53-4$ & 6249 & Ampicillin & Sensitizer & 8 \\
\hline & 2 & $61-33-6$ & 5904 & Benzylpenicillin & Sensitizer & 8 \\
\hline & 3 & $\begin{array}{c}15686-71- \\
2\end{array}$ & 27447 & Cephalexin & Sensitizer & 8 \\
\hline & 4 & $69-52-3$ & 23663979 & Ampicillin & Sensitizer & 8 \\
\hline
\end{tabular}




\begin{tabular}{|c|c|c|c|c|c|c|}
\hline Cluster & $\begin{array}{c}\text { Number } \\
\text { Within } \\
\text { Cluster } \\
\end{array}$ & CASRN & CID & Chemical Name & $\begin{array}{c}\text { Respiratory } \\
\text { Sensitization } \\
\text { Status } \\
\end{array}$ & $\begin{array}{l}\text { Number of } \\
\text { Neighbors }\end{array}$ \\
\hline & 5 & $69-57-8$ & 23668834 & Benzyl penicillin & Sensitizer & 8 \\
\hline & 6 & $\begin{array}{l}\text { 61336-70- } \\
7\end{array}$ & 62883 & Amoxicillin & Sensitizer & 7 \\
\hline & 7 & $\begin{array}{c}26787-78- \\
0 \\
\end{array}$ & 33613 & Amoxicillin & Sensitizer & 7 \\
\hline & 8 & $\begin{array}{c}61477-96- \\
1\end{array}$ & 43672 & Piperacillin & Sensitizer & 6 \\
\hline & 9 & $\begin{array}{l}59703-84- \\
3 \\
\end{array}$ & 23666879 & Piperacillin & Sensitizer & 6 \\
\hline & 10 & $\begin{array}{c}50370-12- \\
2 \\
\end{array}$ & 47965 & Cefadroxil & Sensitizer & 3 \\
\hline & 11 & $\begin{array}{c}66592-87- \\
8 \\
\end{array}$ & 47964 & Cefadroxil & Sensitizer & 3 \\
\hline \multirow{4}{*}{4} & 1 & $110-85-0$ & 4837 & Piperazine & Sensitizer & 3 \\
\hline & 2 & $111-40-0$ & 8111 & Diethylenetriamine & Sensitizer & 3 \\
\hline & 3 & $112-24-3$ & 5565 & Triethylenetetramine & Sensitizer & 2 \\
\hline & 4 & $142-64-3$ & 8893 & Piperazine dihydrochloride & Non-sensitizer & 2 \\
\hline \multirow{4}{*}{5} & 1 & $989-51-5$ & 65064 & Epigallocatechin gallate & Sensitizer & 3 \\
\hline & 2 & $1260-17-9$ & 10255083 & Carminic acid & Sensitizer & 2 \\
\hline & 3 & $\begin{array}{c}16462-65- \\
0 \\
\end{array}$ & 104836 & Plicatic acid & Sensitizer & 2 \\
\hline & 4 & $83-79-4$ & 6758 & Rotenone (ISO) & Non-sensitizer & 1 \\
\hline \multirow{4}{*}{6} & 1 & $111-42-2$ & 8113 & Diethanolamine & Sensitizer & 2 \\
\hline & 2 & $102-71-6$ & 7618 & Triethanolamine & Sensitizer & 2 \\
\hline & 3 & $111-41-1$ & 8112 & Aminoethylethanolamine & Sensitizer & 1 \\
\hline & 4 & $108-01-0$ & 7902 & Dimethyl ethanolamine & Sensitizer & 1 \\
\hline \multirow{4}{*}{7} & 1 & $95-63-6$ & 7247 & 1,2,4-Trimethylbenzene & Non-sensitizer & 3 \\
\hline & 2 & $100-42-5$ & 7501 & Styrene & Sensitizer & 3 \\
\hline & 3 & $108-88-3$ & 1140 & Toluene & Non-sensitizer & 2 \\
\hline & 4 & $92-94-4$ & 7115 & 1,1':4',1'-Terphenyl & Non-sensitizer & 2 \\
\hline \multirow{3}{*}{8} & 1 & $\begin{array}{c}24447-78- \\
7\end{array}$ & 90504 & $\begin{array}{c}\text { Ethoxylated bisphenol A } \\
\text { diacrylate }\end{array}$ & Sensitizer & 2 \\
\hline & 2 & $\begin{array}{c}10402-33- \\
2 \\
\end{array}$ & 25238 & Eugenyl phenylacetate & Non-sensitizer & 1 \\
\hline & 3 & $1675-54-3$ & 2286 & Diglycidyl ether of bisphenol A & Sensitizer & 1 \\
\hline \multirow{3}{*}{9} & 1 & $6622-76-0$ & 5323652 & Methyl tiglate & Non-sensitizer & 2 \\
\hline & 2 & $80-62-6$ & 6658 & Methylmethacrylate & Sensitizer & 1 \\
\hline & 3 & $\begin{array}{c}16930-96- \\
4 \\
\end{array}$ & 637523 & Hexyl tiglate & Non-sensitizer & 1 \\
\hline \multirow{3}{*}{10} & 1 & $111-27-3$ & 8103 & 1-Hexanol & Non-sensitizer & 2 \\
\hline & 2 & $108-93-0$ & 7966 & Cyclohexanol & Non-sensitizer & 1 \\
\hline & 3 & 71-36-3 & 263 & Butan-1-ol & Non-sensitizer & 1 \\
\hline \multirow{2}{*}{$\begin{array}{l}\text { No cluster } \\
\text { identified; }\end{array}$} & 1 & $128-37-0$ & 31404 & 2,6-Di-tert-butyl-p-cresol & Non-sensitizer & 1 \\
\hline & 2 & $89-72-5$ & 6984 & 2-sec-Butylphenol & Non-sensitizer & 1 \\
\hline
\end{tabular}




\begin{tabular}{|c|c|c|c|c|c|c|}
\hline Cluster & $\begin{array}{c}\text { Number } \\
\text { Within } \\
\text { Cluster } \\
\end{array}$ & CASRN & CID & Chemical Name & $\begin{array}{c}\text { Respiratory } \\
\text { Sensitization } \\
\text { Status } \\
\end{array}$ & $\begin{array}{l}\text { Number of } \\
\text { Neighbors }\end{array}$ \\
\hline \multirow{35}{*}{$\begin{array}{l}\text { chemicals } \\
\text { with only } \\
\text { one } \\
\text { neighbor } \\
\text { in } \\
\text { chemical } \\
\text { similarity } \\
\text { map }\end{array}$} & 3 & $94-75-7$ & 1486 & 2,4-Dichlorophenoxyacetic acid & Non-sensitizer & 1 \\
\hline & 4 & $136-78-7$ & 23690431 & $\begin{array}{c}\text { Sodium 2-(2,4- } \\
\text { dichlorophenoxy)ethyl sulphate }\end{array}$ & Non-sensitizer & 1 \\
\hline & 5 & $4442-79-9$ & 20508 & Cyclohexylethyl alcohol & Non-sensitizer & 1 \\
\hline & 6 & $4621-04-9$ & 20739 & p-Isopropylcyclohexanol & Non-sensitizer & 1 \\
\hline & 7 & $97-87-0$ & 7353 & Butyl isobutyrate & Non-sensitizer & 1 \\
\hline & 8 & $106-27-4$ & 7795 & Isoamyl butyrate & Non-sensitizer & 1 \\
\hline & 9 & $79-27-6$ & 6588 & 1,1,2,2-Tetrabromoethane & Non-sensitizer & 1 \\
\hline & 10 & $\begin{array}{c}13171-00- \\
1\end{array}$ & 61585 & $\begin{array}{l}\text { 4-Acetyl-6-tert-butyl-1,1- } \\
\text { dimethylindane }\end{array}$ & Non-sensitizer & 1 \\
\hline & 11 & $106-93-4$ & 7839 & 1,2-Dibromoethane & Non-sensitizer & 1 \\
\hline & 12 & $88-29-9$ & 6930 & Acetyl ethyltetramethyltetralin & Non-sensitizer & 1 \\
\hline & 13 & $1401-69-0$ & 5280440 & Tylosin & Sensitizer & 1 \\
\hline & 14 & $\begin{array}{l}74610-55- \\
2\end{array}$ & 60196281 & Tylosin tartrate & Sensitizer & 1 \\
\hline & 15 & $\begin{array}{l}36519-31- \\
0\end{array}$ & 39859 & Salbutamol & Sensitizer & 1 \\
\hline & 16 & 4098-71-9 & 169132 & Isophorone diisocyanate & Sensitizer & 1 \\
\hline & 17 & $\begin{array}{l}18559-94- \\
9\end{array}$ & 2083 & Salbutamol & Sensitizer & 1 \\
\hline & 18 & $\begin{array}{l}38661-72- \\
2\end{array}$ & 53172 & $\begin{array}{c}1,3- \\
\text { Bis(isocyanatomethyl)cyclohexane }\end{array}$ & Sensitizer & 1 \\
\hline & 19 & $3779-63-3$ & 77411 & $\begin{array}{c}\text { 1,3,5-tris-(6-isocyanato-hexyl)- } \\
\text {,1,3,5.triazinane-2,4,6-trione }\end{array}$ & Sensitizer & 1 \\
\hline & 20 & $7085-85-0$ & 81530 & Ethyl cyanoacrylate & Sensitizer & 1 \\
\hline & 21 & $4035-89-6$ & 19950 & $\begin{array}{l}\text { Biuret of hexamethylene } \\
\text { diisocyanate }\end{array}$ & Sensitizer & 1 \\
\hline & 22 & $137-05-3$ & 8711 & Methyl-2-cyanoacrylate & Sensitizer & 1 \\
\hline & 23 & $2425-06-1$ & 16760159 & Captafol & Sensitizer & 1 \\
\hline & 24 & $133-06-2$ & 18594026 & Captan (ISO) & Non-sensitizer & 1 \\
\hline & 25 & $561-27-3$ & 5462328 & Diacetyl morphine & Sensitizer & 1 \\
\hline & 26 & $52-26-6$ & 5464110 & Morphine hydrochloride & Sensitizer & 1 \\
\hline & 27 & $304-20-1$ & 9351 & Hydralazine & Sensitizer & 1 \\
\hline & 28 & $86-54-4$ & 3637 & Hydralazine & Sensitizer & 1 \\
\hline & 29 & $127-65-1$ & 3641960 & Chloramine- $\mathrm{T}$ & Sensitizer & 1 \\
\hline & 30 & $98-59-9$ & 7397 & p-Toluenesulphonyl chloride & Non-sensitizer & 1 \\
\hline & 31 & $124-04-9$ & 196 & Adipic acid & Sensitizer & 1 \\
\hline & 32 & $693-23-2$ & 12736 & Dodecanedioic acid & Sensitizer & 1 \\
\hline & 33 & $123-31-9$ & 785 & Hydroquinone & Sensitizer & 1 \\
\hline & 34 & $120-80-9$ & 289 & Pyrocatechol & Non-sensitizer & 1 \\
\hline & 35 & $121-25-5$ & 73341 & Amprolium hydrochloride & Sensitizer & 1 \\
\hline & 36 & $137-88-2$ & 8732 & Amprolium hydrochloride & Sensitizer & 1 \\
\hline & 37 & $78-30-8$ & 6527 & Tri-o-tolyl phosphate & Non-sensitizer & 1 \\
\hline
\end{tabular}




\begin{tabular}{|c|c|c|c|c|c|c|}
\hline Cluster & $\begin{array}{l}\text { Number } \\
\text { Within } \\
\text { Cluster }\end{array}$ & CASRN & CID & Chemical Name & $\begin{array}{c}\text { Respiratory } \\
\text { Sensitization } \\
\text { Status }\end{array}$ & $\begin{array}{l}\text { Number of } \\
\text { Neighbors }\end{array}$ \\
\hline & 38 & $115-86-6$ & 8289 & Triphenyl phosphate & Non-sensitizer & 1 \\
\hline & 39 & $110-43-0$ & 8051 & Heptan-2-one & Non-sensitizer & 1 \\
\hline & 40 & $591-78-6$ & 11583 & Hexan-2-one & Non-sensitizer & 1 \\
\hline \multirow{32}{*}{$\begin{array}{c}\text { No } \\
\text { Neighbors } \\
\text { (Not } \\
\text { pictured } \\
\text { in Figure } \\
\text { S2) }\end{array}$} & 1 & $551-16-6$ & 11082 & 6-Amino penicillanic acid & Sensitizer & 0 \\
\hline & 2 & $4424-06-0$ & 78141 & Brilliant orange GR & Sensitizer & 0 \\
\hline & 3 & $55-56-1$ & 2713 & Chlorhexidene & Sensitizer & 0 \\
\hline & 4 & $62-73-7$ & 3039 & Dichlorvos & Sensitizer & 0 \\
\hline & 5 & $101-68-8$ & 7570 & $\begin{array}{l}\text { Diphenyl methane- } 4,4 *_{-} \\
\text {diisocyanate }\end{array}$ & Sensitizer & 0 \\
\hline & 6 & $141-43-5$ & 700 & Ethanolamine & Sensitizer & 0 \\
\hline & 7 & $107-15-3$ & 3301 & Ethylenediamine & Sensitizer & 0 \\
\hline & 8 & $55-38-9$ & 3346 & Fenthion & Sensitizer & 0 \\
\hline & 9 & $822-06-0$ & 13192 & Hexamethylene diisocyanate & Sensitizer & 0 \\
\hline & 10 & $108-31-6$ & 7923 & Maleic anhydride & Sensitizer & 0 \\
\hline & 11 & $555-30-6$ & 38853 & Methyldopa & Sensitizer & 0 \\
\hline & 12 & $1897-45-6$ & 15910 & Tetrachloroisophthalonitrile & Sensitizer & 0 \\
\hline & 13 & $117-08-8$ & 8326 & Tetrachlorophthalic anhydride & Sensitizer & 0 \\
\hline & 14 & $116-66-5$ & 67005 & $\begin{array}{l}\text { 1,1,3,3,5-Pentamethyl-4,6- } \\
\text { Dinitroindane }\end{array}$ & Non-sensitizer & 0 \\
\hline & 15 & $470-67-7$ & & 1,4-Cineole & Non-sensitizer & 0 \\
\hline & 16 & $\begin{array}{l}54546-26- \\
8\end{array}$ & 94569 & $\begin{array}{l}\text { 2-Butyl-4,4,6-trimethyl-1,3- } \\
\text { dioxane }\end{array}$ & Non-sensitizer & 0 \\
\hline & 17 & $\begin{array}{c}\text { 67874-72- } \\
0\end{array}$ & 62240 & 2-tert-Amylcyclohexyl acetate & Non-sensitizer & 0 \\
\hline & 18 & $\begin{array}{c}60763-42- \\
0 \\
\end{array}$ & 108506 & 3,6-Dimethyloctan-3-yl acetate & Non-sensitizer & 0 \\
\hline & 19 & $\begin{array}{l}50816-18- \\
7 \\
\end{array}$ & 39801 & 9-Decenyl acetate & Non-sensitizer & 0 \\
\hline & 20 & $142-19-8$ & 8878 & Allyl heptylate & Non-sensitizer & 0 \\
\hline & 21 & $79-92-5$ & 6616 & Camphene & Non-sensitizer & 0 \\
\hline & 22 & $\begin{array}{c}65405-76- \\
7 \\
\end{array}$ & 5372353 & cis-3-Hexenyl anthranilate & Non-sensitizer & 0 \\
\hline & 23 & $\begin{array}{l}21662-09- \\
9 \\
\end{array}$ & 5362620 & cis-4-Decen-1-al & Non-sensitizer & 0 \\
\hline & 24 & $\begin{array}{c}52671-32- \\
6 \\
\end{array}$ & 40058 & Citronellyl nitrile & Non-sensitizer & 0 \\
\hline & 25 & $544-40-1$ & 11002 & Dibutyl sulfide & Non-sensitizer & 0 \\
\hline & 26 & $95-41-0$ & 66768 & Dihydro-isojasmone & Non-sensitizer & 0 \\
\hline & 27 & $6413-10-1$ & 80865 & $\begin{array}{c}\text { Ethyl acetoacetate ethylene glycol } \\
\text { ketal }\end{array}$ & Non-sensitizer & 0 \\
\hline & 28 & $97-64-3$ & 7344 & Ethyl lactate & Non-sensitizer & 0 \\
\hline & 29 & 2305-05-7 & 16821 & g-Dodecalactone & Non-sensitizer & 0 \\
\hline & 30 & $5870-93-9$ & 62592 & Heptyl butyrate & Non-sensitizer & 0 \\
\hline & 31 & $110-54-3$ & 8058 & Hexane & Non-sensitizer & 0 \\
\hline & 32 & $97-45-0$ & 7336 & 1-Carvyl propionate & Non-sensitizer & 0 \\
\hline
\end{tabular}




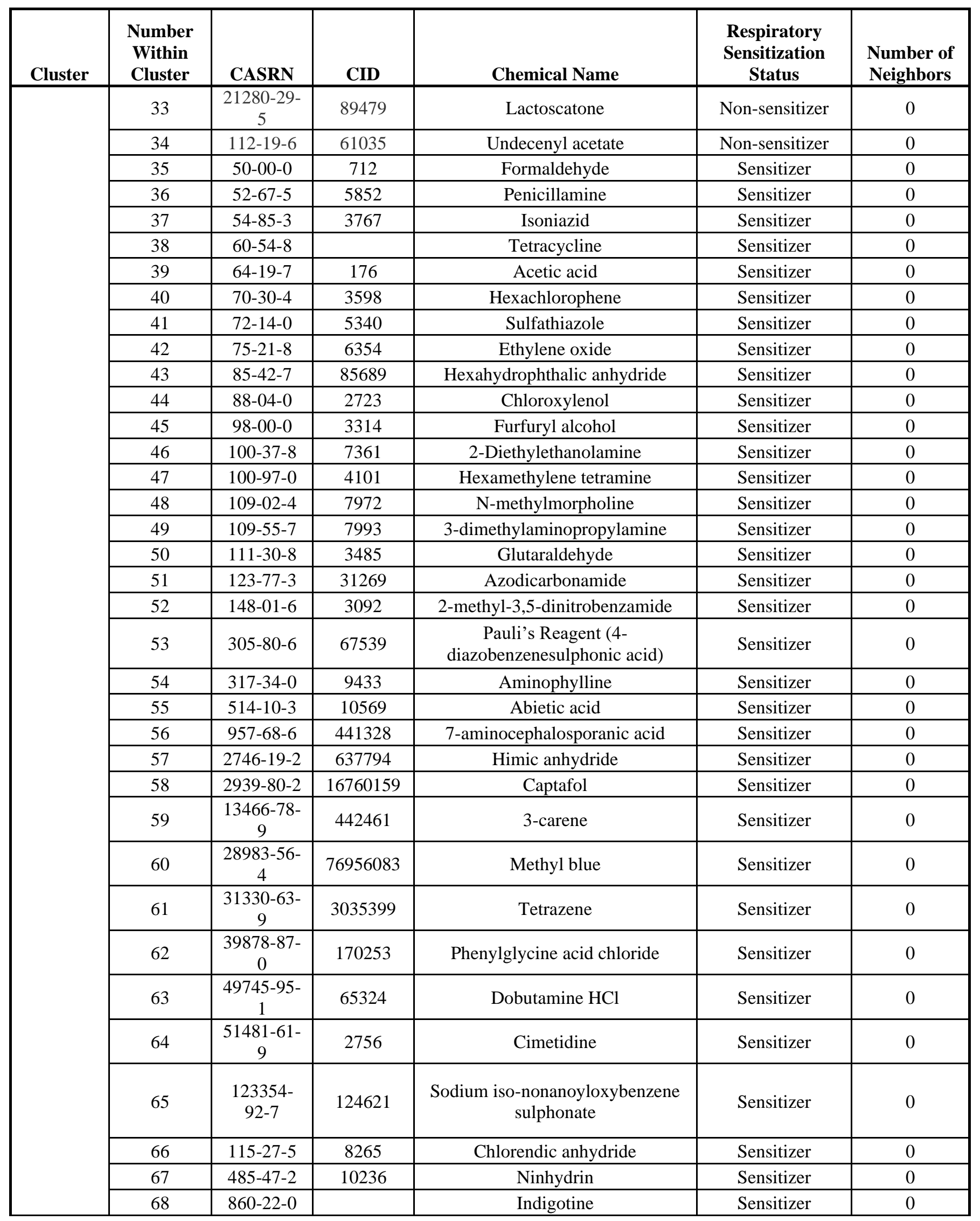




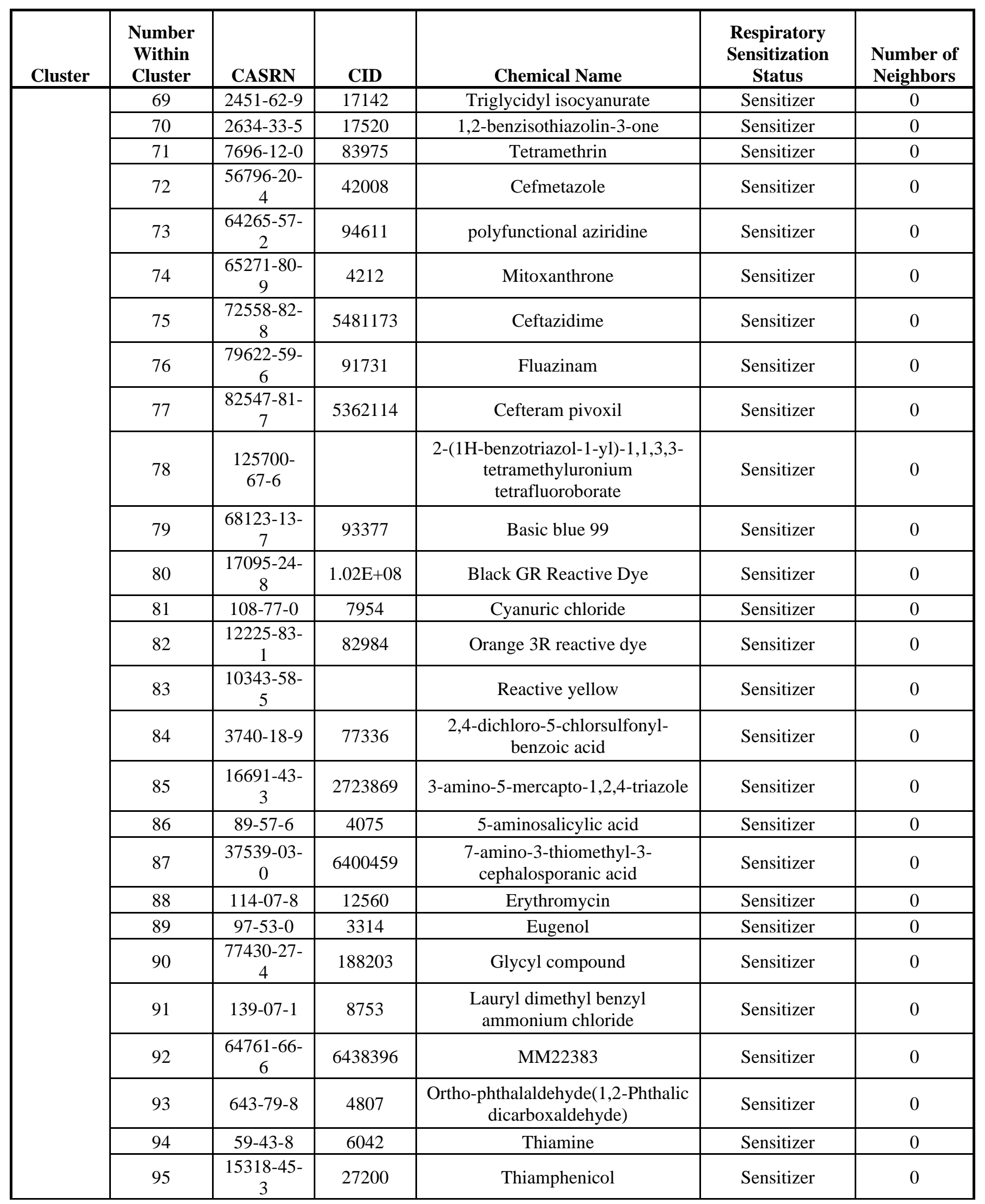




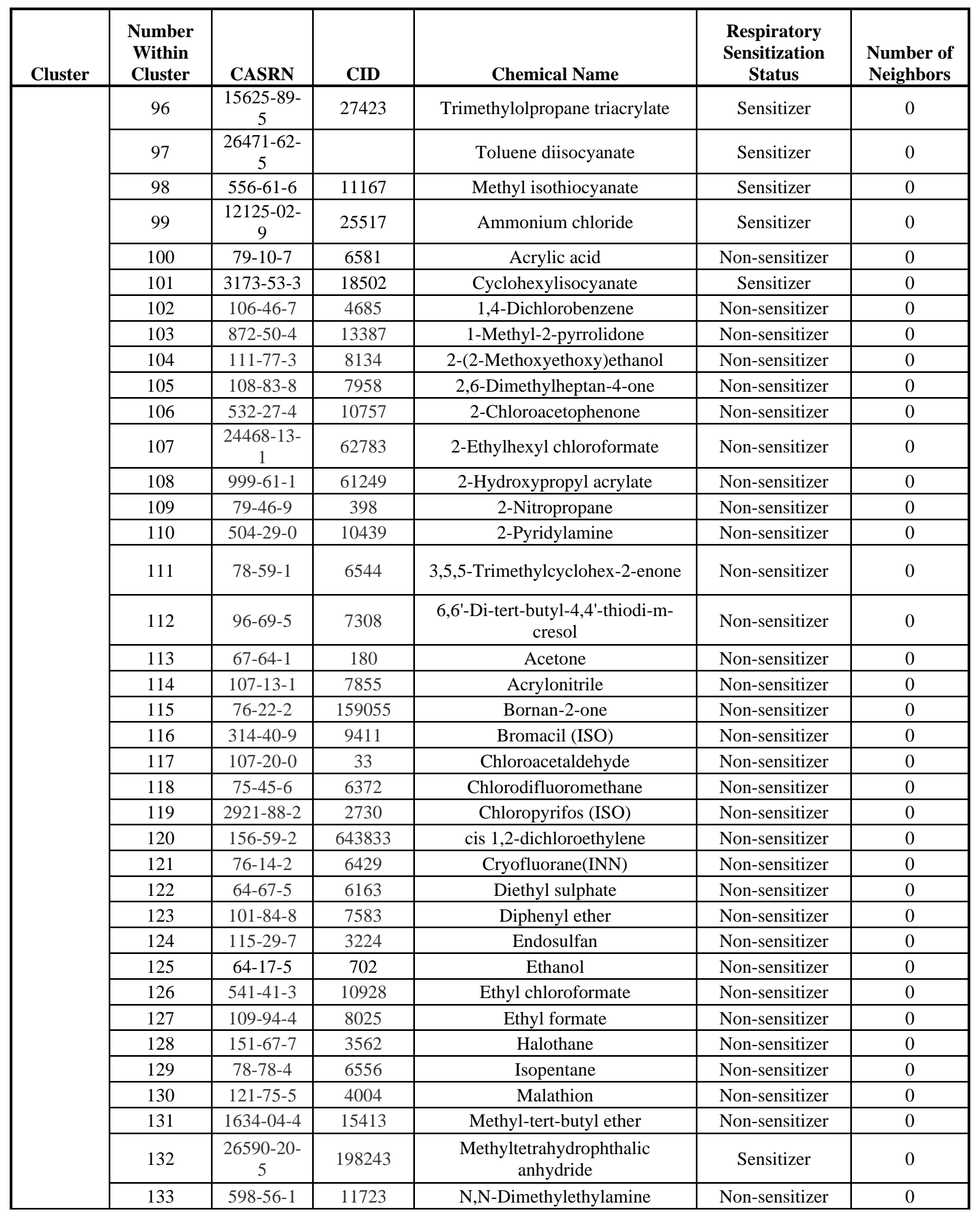




\begin{tabular}{|c|c|c|c|c|c|c|}
\hline Cluster & $\begin{array}{c}\text { Number } \\
\text { Within } \\
\text { Cluster }\end{array}$ & CASRN & CID & Chemical Name & $\begin{array}{c}\text { Respiratory } \\
\text { Sensitization } \\
\text { Status }\end{array}$ & $\begin{array}{c}\text { Number of } \\
\text { Neighbors }\end{array}$ \\
\hline \multirow{6}{*}{134} & $463-82-1$ & 10041 & Neopentane & Non-sensitizer & 0 \\
\cline { 2 - 7 } & 135 & $54-11-5$ & 89594 & Nicotine & Non-sensitizer & 0 \\
\cline { 2 - 7 } & 136 & $144-62-7$ & 971 & Oxalic acid & Non-sensitizer & 0 \\
\cline { 2 - 7 } & 137 & $298-02-2$ & 4790 & Phorate & Non-sensitizer & 0 \\
\cline { 2 - 7 } & 138 & $1918-02-1$ & 15965 & Picloram (ISO) & Non-sensitizer & 0 \\
\cline { 2 - 7 } & 139 & $88-89-1$ & 6954 & Picric acid & Non-sensitizer & 0 \\
\cline { 2 - 7 } & 140 & $114-26-1$ & 4944 & Propoxur & Non-sensitizer & 0 \\
\cline { 2 - 7 } & 141 & $525-66-6$ & 4946 & Propranolol & Non-sensitizer & 0 \\
\cline { 2 - 7 } & 142 & $57-50-1$ & 5988 & Sucrose & Non-sensitizer & 0 \\
\cline { 2 - 7 } & 143 & $2422-88-0$ & 3032425 & $1,1,3-$ Tributylthiourea (TBTU) & Sensitizer & 0 \\
\cline { 2 - 7 } & 144 & $127-18-4$ & 31373 & Tetrachloroethylene & Non-sensitizer & 0 \\
\cline { 2 - 7 } & 145 & $126-73-8$ & 31357 & Tributyl phosphate & Non-sensitizer & 0 \\
\cline { 2 - 7 } & 146 & $76-06-2$ & 6423 & Trichloronitromethane & Non-sensitizer & 0 \\
\hline & 147 & $25656-78-$ & 186099 & Triphenylmethane triisocyanate & Sensitizer & 0 \\
\hline
\end{tabular}

Table S3: Chemical Identifiers by Clusters in the Chemical Similarity Map. Supplemental

Table 3 identifies the chemicals by cluster as displayed in the chemical similarity map in Figure S2. The similarity cutoff for the chemical similarity map in Figure S2 is 80\%. There are 257 discrete chemicals in the overall data set, and, of these, 147 chemicals had no neighbor at an $80 \%$ similarity cutoff. As these chemicals have no neighbors, the singleton chemicals are not displayed in the chemical similarity map in Figure S2. In addition, several of the chemicals only had one neighbor $(n=40)$. While they are displayed on the chemical similarity map, the individual pairs are not identified as individual clusters on the chemical similarity map or in the table; they are simply listed as a cluster of all chemicals with only one neighbor. 


\begin{tabular}{|l|c|c|c|c|c|c|}
\hline \multirow{2}{*}{ Performance Metric } & \multicolumn{2}{|c|}{ Toxtree } & \multicolumn{2}{c|}{$\begin{array}{c}\text { COEH } \\
\text { (Conservative) }\end{array}$} & \multicolumn{2}{c|}{$\begin{array}{c}\text { COEH } \\
\text { (Not Conservative) }\end{array}$} \\
\cline { 2 - 7 } & $\mathbf{n}$ & $\mathbf{\%}$ & $\mathbf{n}$ & $\mathbf{\%}$ & $\mathbf{n}$ & $\mathbf{\%}$ \\
\hline Sensitivity & 104 & 74 & 106 & 78 & 94 & 69 \\
\hline Specificity & 79 & 68 & 30 & 66 & 42 & 84 \\
\hline False Positive & 37 & 32 & 39 & 34 & 19 & 16 \\
\hline False Negative & 37 & 26 & 30 & 22 & 42 & 31 \\
\hline Coverage & 257 & 100 & 252 & 98 & 252 & 98 \\
\hline
\end{tabular}

Table S4: Performance Metrics. The total data set is 257 chemicals. Coverage is defined as the number of chemicals that returned a prediction after inputting all 257 chemicals. COEH assigns 3 different predictions to chemicals: Hazardous; Borderline, may be hazardous; and Low index, may still be hazardous. Chemicals designated as "Hazardous" were always considered to be respiratory sensitizers, and chemicals designated as "Low index; may still be hazardous" were designated as non-respiratory sensitizers. For the COEH (Conservative) approach, we considered chemicals designated "Borderline, may be hazardous" as respiratory sensitizers. For the COEH (Not Conservative) approach, chemicals designated as "Borderline, may be hazardous" as non-respiratory sensitizers. 


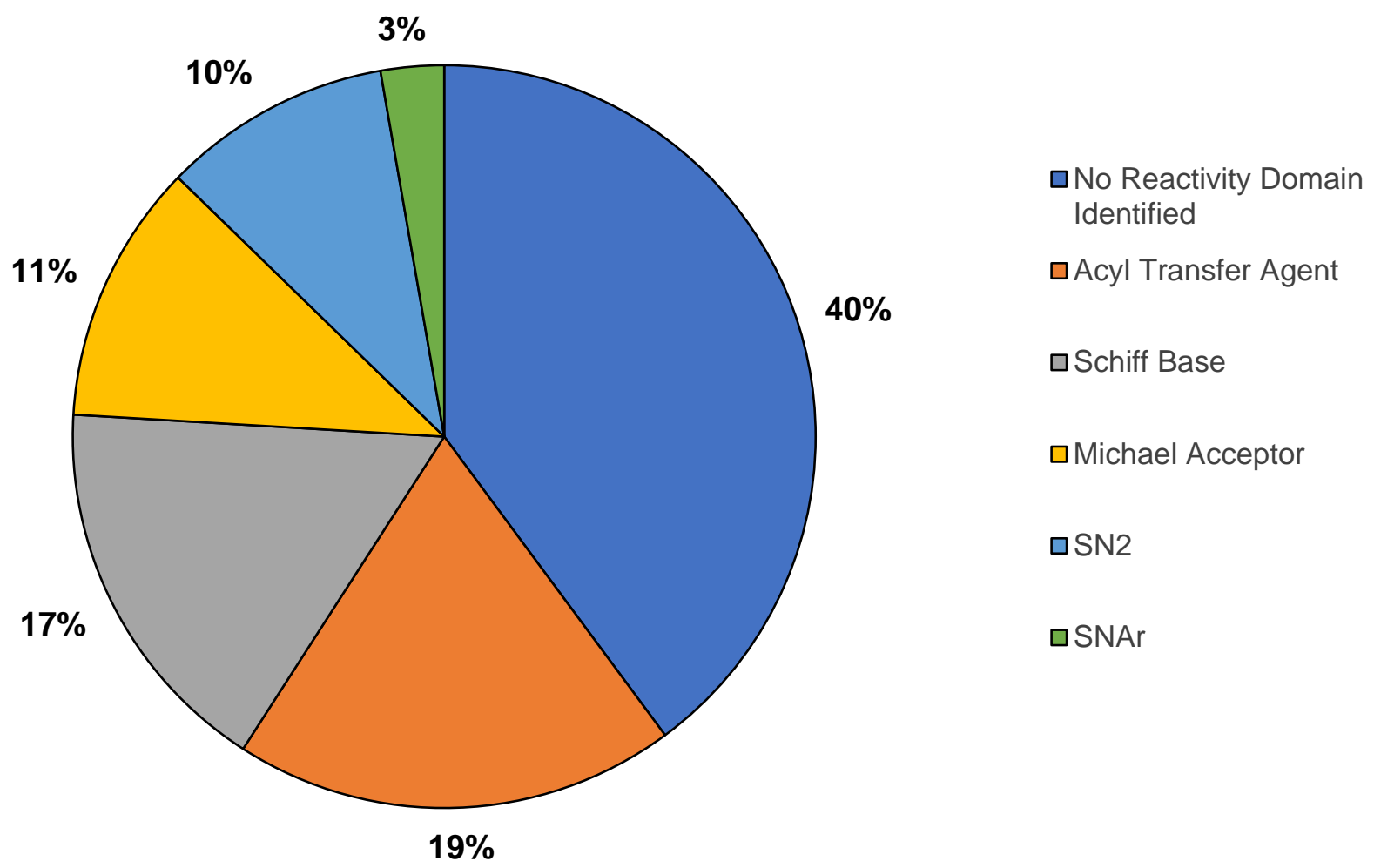

Figure S3: Skin Sensitization Reactivity Alert Distribution. Skin sensitization reactivity domain alerts were assigned using Toxtree for all chemicals in the data set. Some chemicals had more than one alert, and this figure accounts for all alerts from each chemical. 